\title{
Remaining Useful Life Prediction based on Noisy Condition Monitoring Signals using Constrained Kalman Filter
}

\author{
Junbo Son ${ }^{1}$, Shiyu Zhou ${ }^{*}$, Chaitanya Sankavaram ${ }^{2}$, Yilu Zhang ${ }^{2}$, Xinyu Du $^{2}$
}

\begin{abstract}
In this paper, a statistical prognostic method to predict the remaining useful life (RUL) of individual units based on noisy condition monitoring signals is proposed. The prediction accuracy of existing data-driven prognostic methods depends on the capability of accurately modeling the evolution of condition monitoring (CM) signals. Therefore, it is inevitable that the RUL prediction accuracy depends on the amount of random noise in CM signals. When signals are contaminated by a large amount of random noise, RUL prediction even becomes infeasible in some cases. To mitigate this issue, a robust RUL prediction method based on constrained Kalman filter is proposed. The proposed method models the CM signals subject to a set of inequality constraints so that satisfactory prediction accuracy can be achieved regardless of the noise level of signal evolution. The advantageous features of the proposed RUL prediction method is demonstrated by both numerical study and case study with real world data from automotive lead-acid batteries.
\end{abstract}

Index terms: remaining useful life; condition monitoring signals; constrained Kalman filter

\section{Introduction}

Remaining useful life (RUL) prediction is essential to ensure the overall system reliability and to design a successful maintenance strategy. Therefore, significant research efforts have been devoted to RUL prognosis (Si et al. 2011; Gorjian et al. 2010). Moving forward from the traditional time-to-failure analysis, contemporary RUL prognosis emphasizes on the prediction of failure event on an individual unit based on the condition monitoring (CM) signals, also referred as degradation signals in some applications (Zhou et al. 2014). The CM signals are the observable indicators that can be used to infer the unobservable underlying health status of a system, e.g., internal resistance of the automotive battery or bearing vibration measurements of a gearbox. Thus, many prognostic algorithms based on the CM signal observations are proposed recently (Gebraeel et al. 2005; Chen et al. 2013, Sun, Li and Xi 2012; Ye and Chen 2014; Son et al. 2015).

\footnotetext{
${ }^{1}$ Department of Industrial and Systems Engineering, University of Wisconsin-Madison, Madison, WI, 53706, USA

${ }^{*}$ Corresponding Author, e-mail: shiyuzhou@wisc.edu

${ }^{2}$ General Motors Research \& Development, Warren, MI, 48092, USA
} 
The RUL prognostic algorithms that utilize the CM signals can be generally classified into two categories based on the definition of the failure. In the first category, the failure of a system is defined as the time when the CM signal surpasses a pre-specified failure threshold (Lu and Meeker 1993). Often, this type of failure is called the soft failure because the failure is determined by a user-defined threshold (Yu and Fuh 2010). In the second category, the value of the CM signal does not determine the failure occurrence directly, rather, influences the occurrence probability (Liao et al. 2006). This type of failure is called hard failure (Gorjian et al. 2010; Zhou et al. 2014). In both classes of prognostic method, a mixed effects model is typically used to model the evolution of CM signals. Furthermore, to facilitate the accurate RUL prediction for a specific unit, the parameters of the mixed effects model are updated through Bayesian inference framework (Gebraeel et al. 2005; Zhou et al. 2014). Specifically, first, the initial population-level mixed effects model is fitted at the offline stage, which reflects the average behavior of multiple units and provide the prior information. Then, the model updating is conducted in the online stage based on both prior information and the newly collected CM signals from the specific unit to obtain the posterior information. As a result, the posterior contains unique characteristics of the specific unit of our interest. The Bayesian updating has been investigated intensively and it is generally agreed that the model updating is the key for satisfactory RUL prediction accuracy (Son et al. 2013).

Although the mixed effects CM signal model and the online Bayesian model updating have shown its efficacy in various applications, one issue has not been sufficiently addressed in the existing literature. The CM signals (or degradation signals) are indicators of the unobservable health status of a unit or system. Thus, CM signals should be inherently monotonic because the underlying health condition is always deteriorating unless some maintenance actions have been performed (Byon et al. 2010; Zhou et al. 2012). Indeed, most of prognostic methods based on CM signals implicitly assume the monotonicity of the signal. For instance, in the soft failure prognosis, the failure is defined by the time when CM signal passes the failure threshold. Clearly, if the updated signal evolution path shows a decreasing trend in time, the predicted signal would never pass the threshold. Thus, the RUL prediction becomes infeasible (Lim and Mba 2015). Because the signal observations are subject to random disturbances such as the sensor noise, the conventional Bayesian updating method used in the existing literatures cannot guarantee the monotonic trend of the updated CM signal. Recently, recursive filtering methods such as Kalman and particle filters have been actively investigated for the RUL prognosis (Ye et al. (2015); Si et al. (2013); Chen et 
al. (2012); Zio and Peloni (2011)). Those methods are good examples of adopting sequential Bayesian inference to update the CM signal path modeled by, for instance, Weiner process (Ye et al. (2015)). To some extent, stochastic filters can serve as a noise-remover. However, despite of advantageous features, existing filter-based RUL prognostic methods do not have the control over the state space because they are built upon the conventional Bayesian inference framework. Thus, they may still produce counter-intuitive posterior state estimates and hinder reasonable RUL prediction when severe noise is present. This phenomena is illustrated in Figure 1.

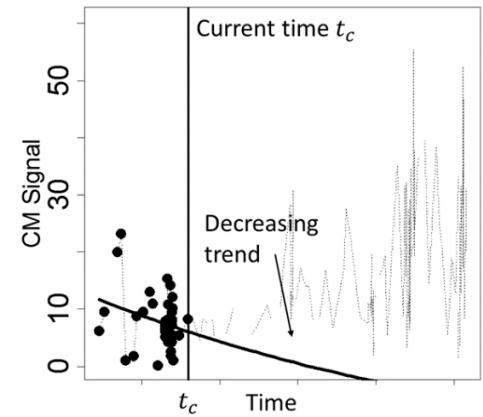

(a) Updated model at $t_{c}$

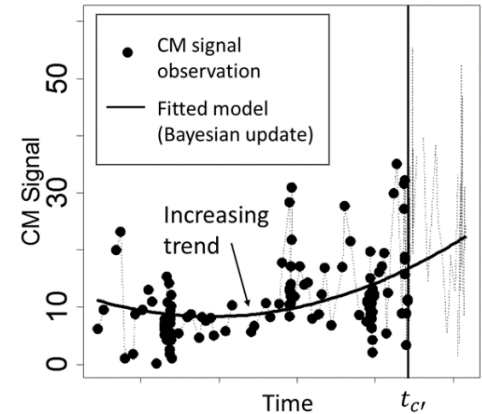

(b) Updated model at $t_{c^{\prime}}>t_{c}$

Figure 1. Illustration of Bayesian updating with violation of monotonicity

Figure 1 shows a CM signal for an automotive lead-acid battery collected from an example unit. From Figure 1(a), the updated model at time $t_{c}$ violates the monotonicity principle and exhibits a decreasing trend in time. The trend becomes increasing when more signal observations are cumulated as shown in Figure 1(b). However, the trend is still not monotonic. Thus, the fitted model based on the conventional Bayesian model updating method does not accurately reflect the true underlying degradation process of the unit.

Even though the abovementioned issue has been recognized in many applications, it has not been thoroughly studied in the existing RUL prognosis literature. Typically, when the CM signals show a high level of noise and the RUL prediction is infeasible, the prediction is skipped at that time and the algorithm waits for more $\mathrm{CM}$ signal observations to accumulate until the prediction becomes feasible again (Lim and Mba 2015). In some cases, the signals with a high level of noise are pre-processed prior to the modeling via signal smoothing techniques such as exponential smoothing (Bluvband, Porotsky, and Tropper 2014). However, these methods do not truly solve the issue and cannot guarantee the monotonicity of the CM signals.

To fill this research gap, we propose an effective online model updating method by using the constrained Kalman filter (CKF) approach. The conventional Bayesian model updating is replaced 
by the CKF procedure so that the undesirable parameter space for the CM signal model can be excluded. In this way, CKF can not only guarantee the monotonicity of the signal but also incorporate specific domain knowledge of the degradation process. To the best of our knowledge, no existing work has been reported on integrating the CKF method into an online failure prognosis framework to deal with the issue of non-monotonicity of the predicted CM signal. A comprehensive simulation study and a real world case study on the RUL prognosis for automotive lead-acid battery demonstrate the effectiveness of the proposed approach. In addition to abovementioned contribution, we also proposed a new method of realizing the CKF algorithm. Among several variants of CKF approaches, the probability density function (PDF) truncation approach is known to be effective for enforcing double-sided inequality constraints (Straka, Dunik, and Simandl 2012). The PDF truncation method truncates the posterior distribution obtained by the conventional unconstrained KF at the edges of the inequality constraints to ensure that the final estimates would lie within the specified constrained space and many promising results have been reported (Shimada et al. 1998). However, the existing PDF truncation methods either depend on a complex variable decomposition (Simon and Simon 2010) or a computationally expensive Monte Carlo approach (Straka, Dunik, and Simandl 2012). Due to such limitations, these methods are less appealing for online real time prognosis. To overcome those limitations and to facilitate efficient online prognosis, we propose a new approach for the PDF truncation by directly using the moment generating function (MGF) that is computationally efficient.

The paper is organized as follows. In Section II, the CM signal model and the corresponding online updating method based on the proposed CKF is introduced. Then, the RUL prognostic framework with CKF is described in Section III. A numerical study that reveals meaningful insights of the proposed method is discussed in Section IV. In Section V, a case study with real world application of RUL prognosis for automotive lead-acid batteries is presented which shows the practical benefit of the proposed method. Lastly, Section VI summarizes the contributions of the proposed method and concludes the paper.

\section{Modeling Noisy CM Signals Based on the Constrained Kalman Filter}

In this section, one of the most popular methods for modeling the CM signal, a mixed effects model, is reviewed. The conventional online Bayesian model updating based on the mixed effects model is presented accordingly. Then, the constrained Kalman filter (CKF) method is described.

\subsection{Review of CM signal models and the online Bayesian model updating}


To model the CM signal evolution, the mixed effects model has been widely used in RUL prognosis literature due to its flexible model structure (Gebraeel et al. 2005). The mixed effects model allows each unit to have its own parameter. In other words, the model assumes that each unit has its own signal propagation path. The mixed effects model for the CM signal evolution is typically defined as (Laird and Ware 1982):

$$
y_{i k}=\theta_{i 1} z_{1}\left(t_{i k}\right)+\cdots+\theta_{i d} z_{d}\left(t_{i k}\right)+\varepsilon_{k}=\boldsymbol{z}_{i k}^{T} \boldsymbol{\theta}_{i}+\varepsilon_{k},
$$

where $y_{i k}$ is the observed CM signal value from the $i$ th unit at $t_{i k}$ (the $k$ th observation time for unit $i), \boldsymbol{\theta}_{i}=\left[\theta_{i 1}, \ldots, \theta_{i d}\right]^{T}$ is the $d$-dimensional mixed effects parameters for the $i$ th unit, $\boldsymbol{z}_{i k}=$ $\left[z_{1}\left(t_{i k}\right), \ldots, z_{d}\left(t_{i k}\right)\right]^{T}$ is the pre-specified time-dependent regression function, and $\varepsilon_{k}$ is the random error term. The time-dependent regression function can be specified freely such as $\boldsymbol{z}_{i k}=$ $\left[1 t_{i k} t_{i k}^{2}\right]^{T}$, i.e., quadratic evolution of the CM signal. The mixed effects can be decomposed into fixed and random effects as $\boldsymbol{\theta}_{i}=\boldsymbol{\mu}+\boldsymbol{u}_{i}$ where $\boldsymbol{\mu}$ is the fixed effect (population-wise average value) and the random effect (unique parameter for individual unit) is denoted as $\boldsymbol{u}_{i} \sim N(\mathbf{0}, \boldsymbol{\Sigma})$. From this formulation, it is easy to see that $\boldsymbol{\theta}_{i} \sim N(\boldsymbol{\mu}, \boldsymbol{\Sigma})$. Also, it is typically assumed that $\varepsilon_{k} \sim N\left(0, \sigma^{2}\right)$, i.e., white noise such as random measurement error introduced by sensors.

To use this model for predicting the future value of the CM signal, the model parameters such as $\boldsymbol{\mu}, \boldsymbol{\Sigma}$, and $\sigma^{2}$ are first estimated in the offline stage based on the CM signals collected from multiple units. Then the estimated model is used as prior and a Bayesian model updating is used to obtain accurate predictions for a specific unit in the online stage. Specifically, the posterior distribution of $\boldsymbol{\theta}_{i}$ based on the newly-observed signal from a specific unit up to the time instant $t_{k}$, i.e., $\boldsymbol{y}_{i, 1: k}=\left[y_{i 1}, \ldots, y_{i k}\right]^{T}$ where $\boldsymbol{y}_{i, 1: k}$ refers to the collection of all CM signal observations from the $i$ th unit up to time index $k$, can be computed as $p\left(\boldsymbol{\theta}_{i} \mid \boldsymbol{y}_{i, 1: k}\right) \propto p\left(\boldsymbol{y}_{i, 1: k} \mid \boldsymbol{\theta}_{i}\right) \pi\left(\boldsymbol{\theta}_{i}\right)$ where $p\left(\boldsymbol{y}_{i, 1: k} \mid \boldsymbol{\theta}_{i}\right)$ denotes the likelihood of the observation vector $\boldsymbol{y}_{i, 1: k}$ and $\pi\left(\boldsymbol{\theta}_{i}\right)$ refers to the prior distribution of $\boldsymbol{\theta}_{i}$ that is obtained from the offline stage. The posterior distribution $p\left(\boldsymbol{\theta}_{i} \mid \boldsymbol{y}_{i, 1: k}\right)$ contains more individual-specific information compared to the prior distribution $\pi\left(\boldsymbol{\theta}_{i}\right)$. Assuming normally-distributed $\boldsymbol{\theta}_{i}$ and $\varepsilon_{k}$, based on the conjugate property, the posterior is also a normal distribution $p\left(\boldsymbol{\theta}_{i} \mid \boldsymbol{y}_{i, 1: k}\right)=N\left(\widehat{\boldsymbol{\mu}}_{i k}, \widehat{\boldsymbol{\Sigma}}_{i k}\right)$ where $\widehat{\boldsymbol{\mu}}_{i k}$ and $\widehat{\boldsymbol{\Sigma}}_{i k}$ respectively indicate the posterior mean and the covariance matrix based on $\boldsymbol{y}_{i, 1: k}$. To distinguish the prior estimates from the offline stage and the updated parameters at the online stage, we shall denote the prior parameter estimates 
as $\boldsymbol{\mu}_{0}$ and $\boldsymbol{\Sigma}_{0}$. It is widely known that the posterior mean and variance in $N\left(\widehat{\boldsymbol{\mu}}_{i k}, \widehat{\boldsymbol{\Sigma}}_{i k}\right)$ has a closedform expression as

$$
\left\{\begin{array}{l}
\widehat{\boldsymbol{\mu}}_{i k}=\widehat{\boldsymbol{\Sigma}}_{i k}\left[\frac{\boldsymbol{Z}_{i 1: k}^{T} \boldsymbol{y}_{i, 1: k}}{\sigma^{2}}+\boldsymbol{\Sigma}_{0}^{-1} \boldsymbol{\mu}_{0}\right] \\
\widehat{\boldsymbol{\Sigma}}_{i k}=\left[\boldsymbol{\Sigma}_{0}^{-1}+\frac{\boldsymbol{Z}_{i 1: k}^{T} \boldsymbol{Z}_{i 1: k}}{\sigma^{2}}\right]^{-1}
\end{array},\right.
$$

where $\boldsymbol{Z}_{i 1: k}=\left[\begin{array}{llll}\boldsymbol{z}_{i 1} & \boldsymbol{z}_{i 2} & \ldots & \boldsymbol{z}_{i k}\end{array}\right]^{T}$ and $\boldsymbol{\mu}_{0}, \boldsymbol{\Sigma}_{0}$, and $\sigma^{2}$ can be replaced by their estimates $\widehat{\boldsymbol{\mu}}_{0}, \widehat{\boldsymbol{\Sigma}}_{0}$, and $\hat{\sigma}^{2}$ from the offline stage. With the updated mean and variance computed by (2), we can predict the future CM signal value of this specific unit for any future time $t>t_{k}$.

\subsection{Kalman filtering formulation for model updating}

The mixed effects model shown in previous section typically assumes normality and linearity. Therefore, under certain model formulations, the Bayesian updating can be directly translated into the popular Kalman filter (KF) approach. Specifically, we convert the mixed effects model in (1) into the state-space model by defining the true underlying mixed effects parameters as the states:

$$
\left\{\begin{array}{l}
\text { State equation: } \boldsymbol{\theta}_{i k}=\boldsymbol{\theta}_{i k-1} \\
\text { Observation equation: } y_{i k}=\boldsymbol{z}_{i k}^{T} \boldsymbol{\theta}_{i k}+\varepsilon_{k}
\end{array}\right.
$$

Using this state-space model formulation, the KF can sequentially estimate the posterior mean $\widehat{\boldsymbol{\mu}}_{i k}$ and variance-covariance $\widehat{\boldsymbol{\Sigma}}_{i k}$ based on the new signal $y_{i k}$ at time index $k$ as:

$$
\begin{aligned}
& \text { Kalman gain: } \boldsymbol{K}_{i k}=\widehat{\boldsymbol{\Sigma}}_{i k-1} \boldsymbol{z}_{i k}\left[\boldsymbol{z}_{i k}^{T} \widehat{\boldsymbol{\Sigma}}_{i k-1} \boldsymbol{z}_{i k}+\sigma^{2}\right]^{-1}, \\
& \text { Updated state estimate: } \widehat{\boldsymbol{\mu}}_{i k}=\widehat{\boldsymbol{\mu}}_{i k-1}+\boldsymbol{K}_{i k}\left(y_{i k}-\boldsymbol{z}_{i k}^{T} \widehat{\boldsymbol{\mu}}_{i k-1}\right), \\
& \text { Updated variance-covariance of state estimate: } \widehat{\boldsymbol{\Sigma}}_{i k}=\left(\mathbf{I}-\boldsymbol{K}_{i k} \boldsymbol{z}_{i k}^{T}\right) \widehat{\boldsymbol{\Sigma}}_{i k-1},
\end{aligned}
$$

where $\widehat{\boldsymbol{\mu}}_{i k-1}$ and $\widehat{\boldsymbol{\Sigma}}_{i k-1}$ are the mean and variance-covariance matrix that are estimated at time index $k-1$. Once a new observation $y_{i k}$ becomes available, the KF updates the state estimate and the covariance by computing the Kalman gain $\boldsymbol{K}_{i k}$. The Kalman gain can be viewed as a weight on the correction term $y_{i k}-\boldsymbol{z}_{i k}^{T} \widehat{\boldsymbol{\theta}}_{i k-1}$ (prediction error) as shown in (5) and $\boldsymbol{K}_{i k}$ is derived by minimizing the mean squared error (Rhodes 1971). It can be shown that the results in (5) and (6) are equivalent to that in (2). Despite of the equivalency between the conventional Bayesian updating and the KF, the KF approach has an advantageous feature especially for the real time prognosis. From (2), we see that the regular Bayesian updating requires a $d$-dimensional matrix inversion $\left[\boldsymbol{\Sigma}_{0}^{-1}+\boldsymbol{Z}_{i 1: k}^{T} \boldsymbol{Z}_{i 1: k} / \sigma^{2}\right]^{-1}$. On the other hand, under the KF formulation, we only need to 
take inverse of a scalar $\left[\mathbf{z}_{i k}^{T} \widehat{\boldsymbol{\Sigma}}_{i k-1} \boldsymbol{z}_{i k}+\sigma^{2}\right]^{-1}$ as in (4) because the dimension of the CM signal $y_{i k}$ is one. This feature is desirable when the mixed effects parameter $\boldsymbol{\theta}_{i}$ has a high dimension.

It should be pointed out that the state-space model in (3) and the corresponding KF formulation in (4)-(6) are different from the KF that is commonly used in engineering practice. For instance, the KF used in signal processing typically assumes that the state is the true signal value clouded by the random measurement noise (Zarchan and Musoff 2005; Lim and Mba 2015). In this case, the KF serves as a noise-remover to extract the true signal value accurately. On the other hand, the KF under our state-space model formulation in (3) tries to estimate the hyper parameters of $\boldsymbol{\theta}_{i}$ in the mixed effects model rather than extracting the true signal value.

\subsection{Constrained Kalman filter using truncated moment generating function}

In this section, we extend the KF so that the parameters are updated according to specified constraints. The KF eventually gives $\widehat{\boldsymbol{\mu}}_{i k}$ and $\widehat{\boldsymbol{\Sigma}}_{i k}$ where $\widehat{\boldsymbol{\mu}}_{i k} \in \mathbb{R}^{d}$. Because the true individual parameter $\boldsymbol{\theta}_{i}$ for a specific unit is unobservable, it should be estimated by the posterior mean $\widehat{\boldsymbol{\mu}}_{i k}$. However, $\widehat{\boldsymbol{\mu}}_{i k}$ could be an unreasonable value which does not make any practical sense as shown by the example in the introduction section. If we knew, from the knowledge of the physical degradation process, that $\boldsymbol{\theta}_{i}$ should be within a specific region $\boldsymbol{\Omega}$, then the KF posterior characterized by $N\left(\widehat{\boldsymbol{\mu}}_{i k}, \widehat{\boldsymbol{\Sigma}}_{i k}\right)$ should be truncated at certain boundaries to ensure $\mathrm{P}\left[\widehat{\boldsymbol{\mu}}_{i k} \in \boldsymbol{\Omega}\right]=1$. This can be achieved through a PDF truncation step in addition to the steps in a regular Kalman filter as illustrated in Figure 2.

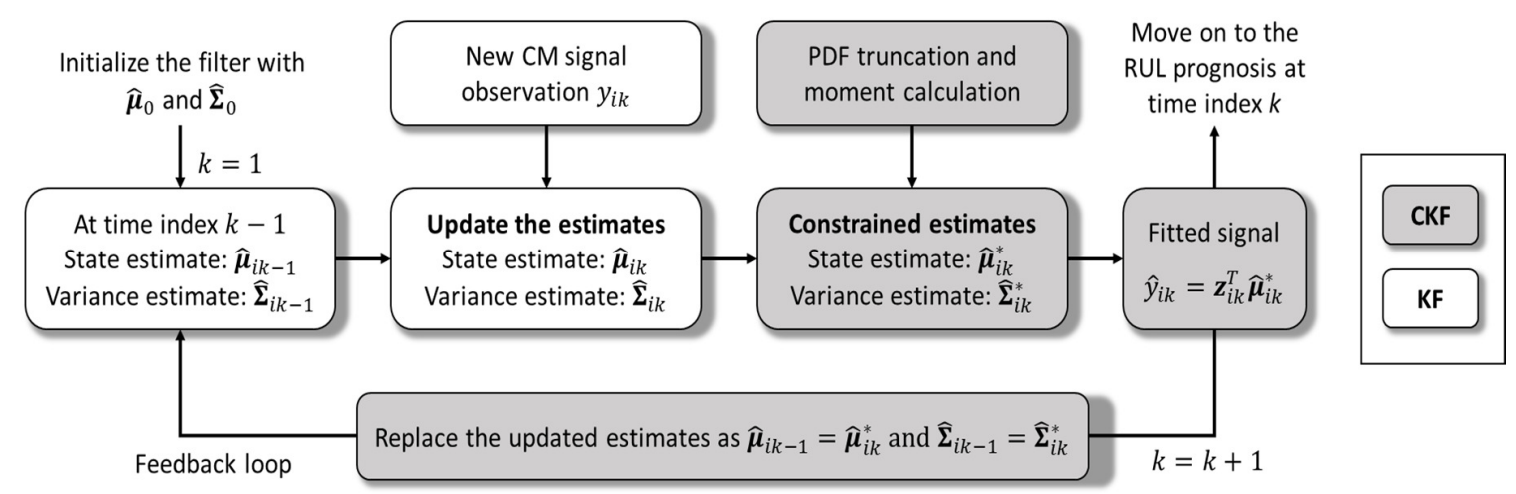

Figure 2. Algorithm summary for the proposed CKF procedure

In more details, suppose we have multiple inequality constraints on the true parameter $\boldsymbol{\theta}_{i}=$ $\left[\theta_{i 1}, \ldots, \theta_{i d}\right]^{T}(d$ number of constraints) as:

$$
a_{j} \leq \theta_{i j} \leq b_{j} \quad j=1, \ldots, d, \forall i,
$$


where $a_{j}<b_{j}$. At time index $k$, we have $\boldsymbol{\theta}_{i} \sim N\left(\widehat{\boldsymbol{\mu}}_{i k}, \widehat{\boldsymbol{\Sigma}}_{i k}\right)$. Thus, enforcing the set of constraints given in (7) yields the truncated multivariate normal distribution denoted as $\operatorname{TN}\left(\widehat{\boldsymbol{\mu}}_{i k}, \widehat{\boldsymbol{\Sigma}}_{i k}, \boldsymbol{a}, \boldsymbol{b}\right)$ where $\boldsymbol{a}=\left\{a_{j}\right\}$ and $\boldsymbol{b}=\left\{b_{j}\right\}$ for $j=1, . ., d$. The PDF for $\operatorname{TN}\left(\widehat{\boldsymbol{\mu}}_{i k}, \widehat{\boldsymbol{\Sigma}}_{i k}, \boldsymbol{a}, \boldsymbol{b}\right)$ is defined as:

$$
f_{T}\left(\boldsymbol{\theta}_{i} ; \widehat{\boldsymbol{\mu}}_{i k}, \widehat{\boldsymbol{\Sigma}}_{i k}, \boldsymbol{a}, \boldsymbol{b}\right)=\frac{f\left(\boldsymbol{\theta}_{i} ; \widehat{\boldsymbol{\mu}}_{i k}, \widehat{\boldsymbol{\Sigma}}_{i k}\right)}{F\left(\boldsymbol{b} ; \widehat{\boldsymbol{\mu}}_{i k}, \widehat{\boldsymbol{\Sigma}}_{i k}\right)-F\left(\boldsymbol{a} ; \widehat{\boldsymbol{\mu}}_{i k}, \widehat{\boldsymbol{\Sigma}}_{i k}\right)} \text { for } a_{j} \leq \theta_{i j} \leq b_{j},
$$

where $f_{T}\left(\boldsymbol{\theta}_{i} ; \widehat{\boldsymbol{\mu}}_{i k}, \widehat{\boldsymbol{\Sigma}}_{i k}, \boldsymbol{a}, \boldsymbol{b}\right)=0$ elsewhere and $f_{T}(\cdot)$ indicates the truncated density. The probability density and cumulative density functions of the multivariate normal distribution are denoted as $f(\cdot)$ and $F(\cdot)$, respectively. We shall denote the mean and variance-covariance of $\operatorname{TN}\left(\widehat{\boldsymbol{\mu}}_{i k}, \widehat{\boldsymbol{\Sigma}}_{i k}, \boldsymbol{a}, \boldsymbol{b}\right)$ as $\widehat{\boldsymbol{\mu}}_{i k}^{*}$ and $\widehat{\boldsymbol{\Sigma}}_{i k}^{*}$, i.e., truncated mean and truncated covariance matrix. We would like to point out that the boundaries for the inequality constraints $\boldsymbol{a}$ and $\boldsymbol{b}$ can be obtained by various ways. First, in some cases, there exists a physical model that clearly explains the possible evolution paths of a CM signal. If such physical model exists, we can specify $\boldsymbol{a}$ and $\boldsymbol{b}$ accordingly. Second, experts who have been monitoring the system for a long time typically have trustworthy knowledge on the system degradation which could be translated into an empirical confidence band for the possible signal evolution paths. Then, we can convert the empirical confidence bands into the form of constraints by properly specifying $\boldsymbol{a}$ and $\boldsymbol{b}$.

There are multiple strategies to enforce a set of equality or inequality constraints to the KF (Simon and Chia 2002; Simon and Simon 2005; Simon 2010). Here, we focus on the PDF truncation method because it is convenient for enforcing a double-sided inequality constraints (Simon and Simon 2010). As Shimada et al. (1998) have pointed out, the PDF truncation is challenging when the dimension of the system state is larger than one because the moments of the truncated PDF that are required in the CKF scheme are generally intractable. As a result, the PDF truncation methods in existing literature typically use Monte Carlo techniques to compute the necessary moments (Straka, Dunik, and Simandl 2012). However, to compute the moments with satisfactory accuracy, we need large amount of random samples and it inevitably creates heavy computational burden, which is undesirable for the online real time prognosis. More sophisticated approach using Jordan canonical decomposition and Gram-Schmidt orthogonalization is proposed (Simon and Simon 2010) that can reduce the computational burden significantly. This method decouples the multi-dimensional random variable into multiple one dimensional random variables and sequentially enforces the constraints one dimension at a time. By doing so, the computation 
can be remarkably simplified. However, because it enforces the constraints sequentially, the order of constraint enforcement often affects the final results (Simon and Simon 2010).

To overcome these limitations, we propose a new PDF truncation method based on the moment generating function (MGF). Based on the MGF of high-dimensional truncated normal distributions (Manjunath and Wilhelm 2012), our main result can be summarized as follows:

Result: The mean $\widehat{\boldsymbol{\mu}}_{i k}^{*}=\left[\hat{\mu}_{i k, 1}^{*}, \ldots, \hat{\mu}_{i k, d}^{*}\right]^{T}$ and the covariance matrix $\widehat{\operatorname{Cov}^{*}}\left[\theta_{i, j}, \theta_{i, p}\right]$ for $j, p \in$ $\{1, \ldots, d\}$ of a multivariate truncated normal distribution, whose density function is given in (8), can be computed as

$$
\hat{\mu}_{i k, j}^{*}=\hat{\mu}_{i k, j}+\psi_{j}\left(\boldsymbol{a}, \boldsymbol{b} ; \widehat{\boldsymbol{\Sigma}}_{i k}\right)=\hat{\mu}_{i k, j}+\sum_{p=1}^{d} \sigma_{i k, j, p}\left\{\varphi_{p}\left(a_{p}\right)-\varphi_{p}\left(b_{p}\right)\right\},
$$

and

$$
\widehat{\operatorname{Cov}}^{*}\left[\theta_{i, j}, \theta_{i, p}\right]=\sigma_{i k, j, p}+\xi_{j, p}\left(\boldsymbol{a}, \boldsymbol{b} ; \widehat{\boldsymbol{\Sigma}}_{i k}\right)+\eta_{j, p}\left(\boldsymbol{a}, \boldsymbol{b} ; \widehat{\boldsymbol{\Sigma}}_{i k}\right)-\psi_{j}\left(\boldsymbol{a}, \boldsymbol{b} ; \widehat{\boldsymbol{\Sigma}}_{i k}\right) \psi_{p}\left(\boldsymbol{a}, \boldsymbol{b} ; \widehat{\boldsymbol{\Sigma}}_{i k}\right),
$$

where $\sigma_{i k, j, p}$ denotes covariance between the $j$ th and the $p$ th parameters, i.e., the element located at the cross-section between the $j$ th row and the $p$ th column in $\widehat{\boldsymbol{\Sigma}}_{i k}$. The $\psi_{j}\left(\boldsymbol{a}, \boldsymbol{b} ; \widehat{\boldsymbol{\Sigma}}_{i k}\right)$ function is defined in (9) whereas $\xi_{j, p}\left(\boldsymbol{a}, \boldsymbol{b} ; \widehat{\boldsymbol{\Sigma}}_{i k}\right)$ and $\eta_{j, p}\left(\boldsymbol{a}, \boldsymbol{b} ; \widehat{\boldsymbol{\Sigma}}_{i k}\right)$ functions are respectively defined as

$$
\xi_{j, p}\left(\boldsymbol{a}, \boldsymbol{b} ; \widehat{\boldsymbol{\Sigma}}_{i k}\right)=\sum_{q=1}^{d} \frac{\sigma_{i k, j, q} \sigma_{i k, p, q}\left\{a_{q} \varphi_{q}\left(a_{q}\right)-b_{q} \varphi_{q}\left(b_{q}\right)\right\}}{\sigma_{i k, q, q}},
$$

and

$$
\begin{aligned}
\eta_{j, p}\left(\boldsymbol{a}, \boldsymbol{b} ; \widehat{\boldsymbol{\Sigma}}_{i k}\right)=\sum_{q=1}^{d} \sigma_{i k, j, q} & \sum_{q \neq l}\left[\left(\sigma_{i k, p, l}-\frac{\sigma_{i k, q, l} \sigma_{i k, p, q}}{\sigma_{i k, q, q}}\right)\right. \\
& \left.\times\left\{\varphi_{q, l}\left(a_{q}, a_{l}\right)+\varphi_{q, l}\left(b_{q}, b_{l}\right)-\varphi_{q, l}\left(a_{q}, b_{l}\right)-\varphi_{q, l}\left(b_{q}, a_{l}\right)\right\}\right] .
\end{aligned}
$$

In (9) and (10), $\varphi_{p}$ and $\varphi_{q, l}$ are two marginal density functions. The univariate marginal density is $\varphi_{p}(x)=\int_{\boldsymbol{\Omega}_{-p}} f_{T}\left(x, \boldsymbol{\theta}_{-p}\right) d \boldsymbol{\theta}_{-p}$ where $\boldsymbol{\theta}_{-p}$ is a $(d-1)$-dimensional vector that excludes $\theta_{i, p}$, i.e., $\boldsymbol{\theta}_{-p}=\left[\theta_{i, 1}, \ldots, \theta_{i, p-1}, \theta_{i, p+1}, \ldots, \theta_{i, d}\right]^{T}$, and $\boldsymbol{\Omega}_{-p}$ is the constrained parameter space without $\theta_{i, p}$, i.e., $\boldsymbol{\Omega}_{-p}=\left\{a_{j} \leq \theta_{i, j} \leq b_{j}: \forall j \neq p, j=1, \ldots, d\right\}$. The bivariate marginal density is $\varphi_{q, l}(x, y)=$ $\int_{\boldsymbol{\Omega}_{-q-l}} f_{T}\left(x, y, \boldsymbol{\theta}_{-q-l}\right) d \boldsymbol{\theta}_{-q-l}$ where $\boldsymbol{\theta}_{-q-l}$ is a $(d-2)$-dimensional vector $\boldsymbol{\theta}_{-q-l}=$ 
$\left[\theta_{i, 1}, \ldots, \theta_{i, q-1}, \theta_{i, q+1}, \ldots, \theta_{i, l-1}, \theta_{i, l+1}, \ldots, \theta_{i, d}\right]^{T}$ that excludes both $\theta_{i, q}$ and $\theta_{i, l}$ for $q \neq l$, and $\boldsymbol{\Omega}_{-q-l}=\left\{a_{j} \leq \theta_{i, j} \leq b_{j}: \forall j \neq q\right.$ and $\left.\forall j \neq l, j=1, \ldots, d\right\}$ for $q \neq l$.

The proof of this result is given in Appendix. The computational challenge in (9) comes from the evaluation of $\varphi_{p}(x)$. For the one-dimensional marginal truncated normal density, an efficient computing method is readily available in Cartinhour (1990). By computing (9) for every $j, \widehat{\boldsymbol{\mu}}_{i k}^{*}$ can be obtained and treated as the constrained estimate. In (10), the covariance matrix involves not only $\varphi_{p}(x)$ but also $\varphi_{q, l}(x, y)$. Although the computation for the bivariate marginal density is more complex compared to the one-dimensional marginal density $\varphi_{p}(x)$, an efficient algorithm is available for computing $\varphi_{q, l}(x, y)$ for both single-sided (Tallis 1961; Leppard and Tallis 1989) and double-sided constraints (Manjunath and Wilhelm 2012). Based on (9), we can see that, for a finite variance of the random noise $\sigma^{2}<\infty, \hat{\mu}_{i k, j}^{*} \rightarrow \hat{\mu}_{i k, j}$ as $k \rightarrow \infty$ because $\psi_{j}\left(\boldsymbol{a}, \boldsymbol{b} ; \widehat{\boldsymbol{\Sigma}}_{i k}\right) \rightarrow 0$. Thus, many advantageous features of the standard KF asymptotically hold for CKF.
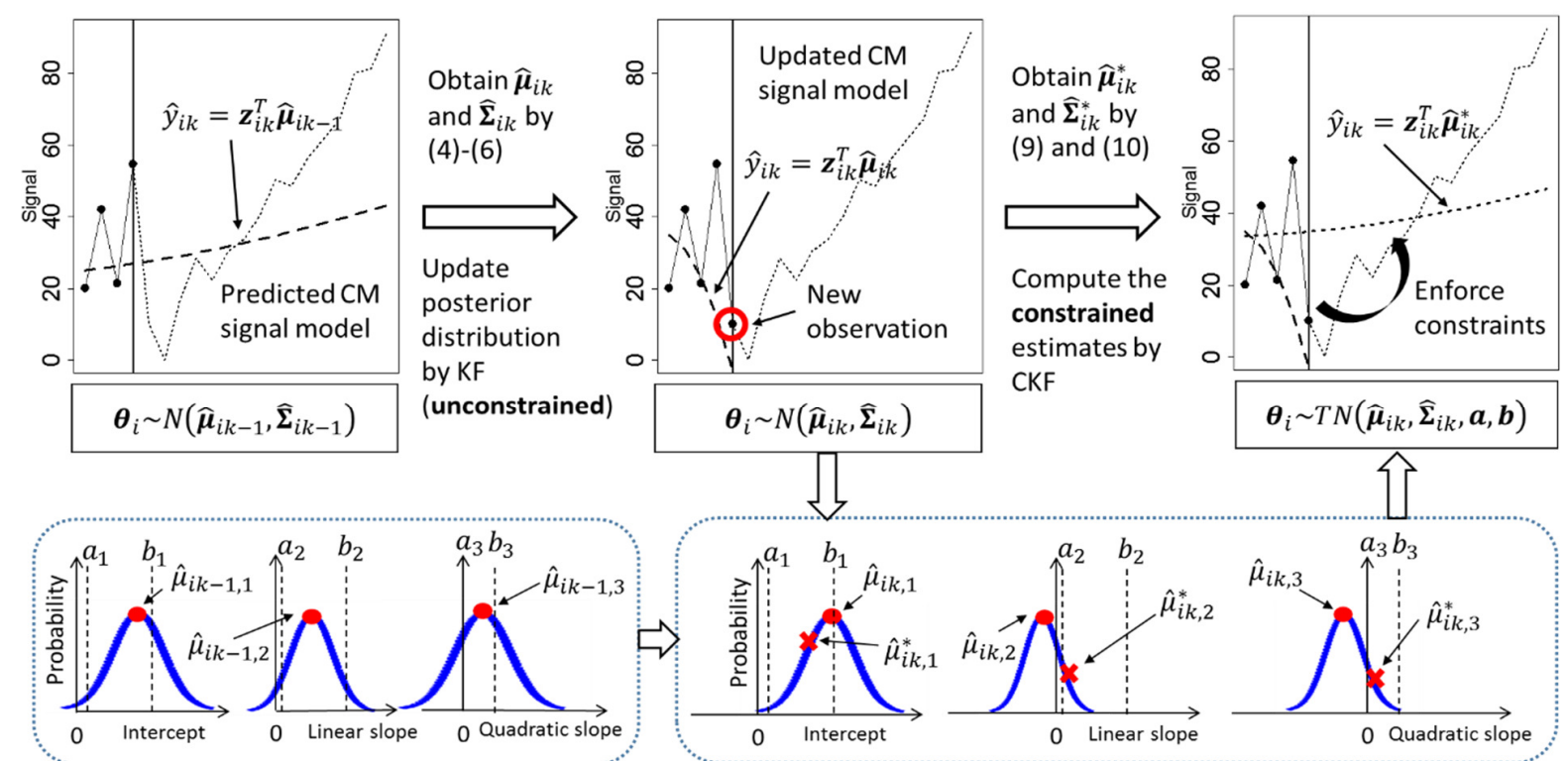

Prior marginal densities

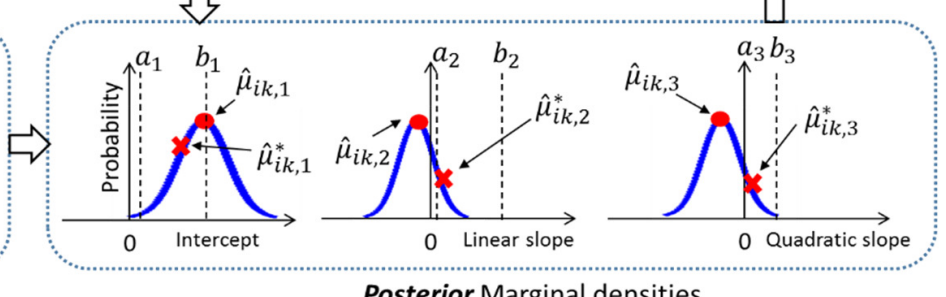

Posterior Marginal densities

Figure 3. Illustration of the proposed CKF method

As a summary, Figure 3 illustrates the overall procedure of the proposed CKF. At each time step $k$, instead of providing $\widehat{\boldsymbol{\mu}}_{i k}$ and $\widehat{\boldsymbol{\Sigma}}_{i k}$ to the KF recursion for the next time instant $k+1$, the CKF gives $\widehat{\boldsymbol{\mu}}_{i k}^{*}$ and $\widehat{\boldsymbol{\Sigma}}_{i k}^{*}$ (truncated moments) which are supposed to be better estimators than $\widehat{\boldsymbol{\mu}}_{i k}$ and $\widehat{\boldsymbol{\Sigma}}_{i k}$ if the constrained space $\boldsymbol{\Omega}$ is properly defined. 


\section{RUL Prediction Based on the Proposed CKF Method}

The proposed $\mathrm{CKF}$ method provides a prediction for the future $\mathrm{CM}$ signal values that guarantees the monotonicity. In principle, it can be integrated into any RUL prognostic framework that requires the forecasting for $\mathrm{CM}$ signals. Without the loss of generality, we illustrate the application of the proposed CKF method in the joint prognostic model (JPM) framework. The JPM is a recently developed method designed to handle the hard failure prognosis and, compared with conventional first-hitting-time prognosis (soft failure), the JPM has desirable properties in some cases. First, when there exist more than a single CM signal, the JPM is preferred because multiple signals and their interactions can be easily accommodated into the JPM structure. Second, when there is no threshold on the CM signal that physically defines the failure of systems, the JPM has shown better prediction accuracy (Son et al. (2013)). Although the technical details of JPM can be found in Zhou et al. (2014), for the sake of completeness, the model structure, estimation, and online prognosis using JPM are briefly reviewed as follows.

\subsection{JPM model structure}

The JPM has its overall model structure defined as

$$
\begin{gathered}
h_{i}(t)=h_{0}(t) \exp \left[\sum_{w=1}^{W} \beta_{w} g_{w}\left(\hat{y}_{i}^{r}(t)\right)\right] \text { for } r=1, \ldots, R \text { and } w=1, \ldots, W, \\
y_{i}^{r}(t)=\hat{y}_{i}^{r}(t)+\varepsilon_{i}^{r}(t)=\left[\mathbf{z}_{i}^{r}(t)\right]^{T} \boldsymbol{\theta}_{i}^{r}+\varepsilon_{i}^{r}(t) \text { for } r=1, \ldots, R,
\end{gathered}
$$

where $h_{i}(t)$ is the unit-specific instantaneous hazard defined as $h_{i}(t)=\lim _{\kappa \rightarrow 0}\left[P\left(t \leq T_{i}<t+\right.\right.$ $\left.\left.\kappa \mid T_{i} \geq t\right) / \kappa\right]$ where $T_{i}$ is the failure time for unit $i, h_{0}(t)$ is the baseline hazard, and $g_{w}(\cdot)$ is a generic function of time-dependent covariate $\hat{y}_{i}^{r}(t)$ for $r=1, \ldots, R$, i.e., $R$ different CM signals. As we can see from (14), each CM signal $y_{i}^{r}(t)$ is modeled by the mixed effects model where the time-dependent regression function $\boldsymbol{z}_{i}^{r}(t)$, the individual mixed effects parameter $\boldsymbol{\theta}_{i}^{r}$, and the random error $\varepsilon_{i}^{r}(t)$ could be different for each CM signal. Based on the fitted CM signals $\hat{y}_{i}^{r}(t)$, the proportional hazards model takes $g_{w}\left(\left[\mathbf{z}_{i}^{r}(t)\right]^{T} \boldsymbol{\theta}_{i}^{r}\right)$ as its time-dependent covariates with associated coefficient $\beta_{w}$. Because $g_{w}(\cdot)$ could be any functions, the time-dependent covariates are typically the CM signal itself and/or some interaction effects of a subset of CM signals.

For the illustration purpose, in this paper, we assume that we have two CM signals $x$ and $y$. Then the JPM model structure can be defined as 


$$
h_{i}(t)=h_{0}(t) \exp \left\{\beta_{0}\left[\mathbf{z}_{i}^{x}(t)\right]^{T} \boldsymbol{\theta}_{i}^{x}+\beta_{1}\left[\mathbf{z}_{i}^{y}(t)\right]^{T} \boldsymbol{\theta}_{i}^{y}+\beta_{2}\left[\mathbf{z}_{i}^{x}(t)\right]^{T} \boldsymbol{\theta}_{i}^{x}\left[\mathbf{z}_{i}^{y}(t)\right]^{T} \boldsymbol{\theta}_{i}^{y}\right\},
$$

where both signals are modeled by mixed effects model as $x_{i}(t)=\left[\mathbf{z}_{i}^{x}(t)\right]^{T} \boldsymbol{\theta}_{i}^{x}+\varepsilon^{x}$ and $y_{i}(t)=$ $\left[\mathbf{z}_{i}^{y}(t)\right]^{T} \boldsymbol{\theta}_{i}^{y}+\varepsilon^{y}$ with random noises $\varepsilon^{x} \sim N\left(0, \sigma_{x}^{2}\right)$ and $\varepsilon^{y} \sim N\left(0, \sigma_{y}^{2}\right)$, respectively. The terms $\boldsymbol{\theta}_{i}^{x}$ and $\boldsymbol{\theta}_{i}^{y}$ are the model parameters of signals $x$ and $y$ for a specific unit $i$ defined as $\boldsymbol{\theta}_{i}^{x} \sim N\left(\boldsymbol{\mu}_{0}^{x}, \boldsymbol{\Sigma}_{0}^{x}\right)$ and $\boldsymbol{\theta}_{i}^{y} \sim N\left(\boldsymbol{\mu}_{0}^{y}, \boldsymbol{\Sigma}_{0}^{y}\right)$. The time-dependent regression functions are denoted as $\boldsymbol{z}_{i}^{x}(t)$ and $\boldsymbol{z}_{i}^{y}(t)$. Two CM signals are respectively linked to the hazard by the coefficients $\beta_{0}$ and $\beta_{1}$. In addition, the interaction effect is included with corresponding coefficient $\beta_{2}$. The coefficients in (15) can be collectively denoted as $\boldsymbol{\beta}=\left\{\beta_{0}, \beta_{1}, \beta_{2}\right\}$. It should be noted that $\boldsymbol{\beta}$ and $h_{0}(t)$ do not have subscription $i$. In other words, those parameters represent the population-wise behavior whereas unit-specific information are contained in $\boldsymbol{\theta}_{i}^{x}$ and $\boldsymbol{\theta}_{i}^{y}$. Overall, the parameters that need to be estimated from data can be denoted as $\boldsymbol{\Theta}=\left\{\boldsymbol{\mu}_{x}, \boldsymbol{\Sigma}_{x}, \boldsymbol{\mu}_{y}, \boldsymbol{\Sigma}_{y}, \sigma_{x}^{2}, \sigma_{y}^{2}, h_{0}(t), \boldsymbol{\beta}\right\}$. Further, we can separate the parameters as $\boldsymbol{\Theta}_{1}=\left\{\boldsymbol{\mu}_{x}, \boldsymbol{\Sigma}_{x}, \boldsymbol{\mu}_{y}, \boldsymbol{\Sigma}_{y}, \sigma_{x}^{2}, \sigma_{y}^{2}\right\}$ and $\boldsymbol{\Theta}_{2}=\left\{h_{0}(t), \boldsymbol{\beta}\right\}$, i.e., $\boldsymbol{\Theta}=\left\{\boldsymbol{\Theta}_{1}, \boldsymbol{\Theta}_{2}\right\}$.

To obtain the parameter estimates $\widehat{\boldsymbol{\Theta}}=\left\{\widehat{\boldsymbol{\Theta}}_{1}, \widehat{\boldsymbol{\Theta}}_{2}\right\}$, there are two approaches well-accepted in the literature. First method is the conventional maximum likelihood estimation theory (Tsiatis, DeGruttola, and Wulfsohn 1995). However, for this method, the computation is very heavy because the full joint likelihood of (15) has very complex form with multiple layers of numerical integration due to the model hierarchy. To overcome this issue, an approximated inference technique called two-stage estimation is widely used (Tsiatis, DeGruttola, and Wulfsohn 1995; Yu and Fuh 2010; Zhou et al. 2014). The two-stage estimation separates the inference in two stages. First, it estimates the parameters for the CM signal model $\widehat{\Theta}_{1}$ and, secondly, it estimates the parameters for the proportional hazards model $\widehat{\boldsymbol{\Theta}}_{2}$. In other words, $\boldsymbol{\Theta}_{2}$ is estimated based on $\widehat{\boldsymbol{\Theta}}_{1}$ as if the fitted CM signals were the true signals. By doing so, we introduce some bias. However, the bias is known to be negligible (Bycott and Taylor 1998; Wulfsohn and Tsiatis 1997) whereas the computational cost reduces substantially. It should be noted that, for the proportional hazards model, $\hat{h}_{0}(t)$ is typically interpolated by non-parametric methods as shown in Zhou et al. (2014).

\subsection{Online RUL prediction}

Based on the estimated parameters of JPM, RUL prediction can be achieved for a specific inservice unit during the online stage. Suppose we have observations from a newly installed unit, say $\tau$, up to the current time index $c$ i.e., $t_{c}$. The survival function for unit $\tau$ can be expressed as 


$$
\begin{aligned}
& S_{\tau}\left(t \mid t_{c}, \boldsymbol{\theta}_{\tau}^{x}, \boldsymbol{\theta}_{\tau}^{y}\right) \\
& =\exp \left\{-\int_{t_{c}}^{t} \hat{h}_{0}(u) \exp \left[\hat{\beta}_{0}\left[\mathbf{z}_{\tau}^{x}(u)\right]^{T} \boldsymbol{\theta}_{\tau}^{x}+\hat{\beta}_{1}\left[\mathbf{z}_{\tau}^{y}(u)\right]^{T} \boldsymbol{\theta}_{\tau}^{y}+\hat{\beta}_{2}\left[\mathbf{z}_{\tau}^{x}(u)\right]^{T} \boldsymbol{\theta}_{\tau}^{x}\left[\mathbf{z}_{\tau}^{y}(u)\right]^{T} \boldsymbol{\theta}_{\tau}^{y}\right] d u\right\},
\end{aligned}
$$

where $t \geq t_{c}$. Based on the collected CM signal observations from the beginning to the current time instant $t_{c}$, we can obtain $f_{T}\left(\boldsymbol{\theta}_{\tau}^{x} ; \widehat{\boldsymbol{\mu}}_{\tau c}^{x}, \widehat{\boldsymbol{\Sigma}}_{\tau c}^{x}, \boldsymbol{a}_{x}, \boldsymbol{b}_{x}\right)$ and $f_{T}\left(\boldsymbol{\theta}_{\tau}^{y} ; \widehat{\boldsymbol{\mu}}_{\tau c}^{y}, \widehat{\boldsymbol{\Sigma}}_{\tau c}^{y}, \boldsymbol{a}_{y}, \boldsymbol{b}_{y}\right)$ from the CKF where $\left\{\boldsymbol{a}_{x}, \boldsymbol{b}_{x}\right\}$ and $\left\{\boldsymbol{a}_{y}, \boldsymbol{b}_{y}\right\}$ define the inequality constraints for signal $x$ and $y$, respectively. Then, the survival function for unit $\tau$ integrating out $\boldsymbol{\theta}_{\tau}^{x}$ and $\boldsymbol{\theta}_{\tau}^{y}$ can be expressed as

$$
\begin{aligned}
& S_{\tau}\left(t \mid t_{c}, \boldsymbol{x}_{\tau, 1: c}, \boldsymbol{y}_{\tau, 1: c}\right) \\
& =\int S\left(t \mid t_{c}, \boldsymbol{\theta}_{\tau}^{x}, \boldsymbol{\theta}_{\tau}^{y}\right) f_{T}\left(\boldsymbol{\theta}_{\tau}^{x} ; \widehat{\boldsymbol{\mu}}_{\tau c}^{x}, \widehat{\boldsymbol{\Sigma}}_{\tau c}^{x}, \boldsymbol{a}_{x}, \boldsymbol{b}_{x}\right) f_{T}\left(\boldsymbol{\theta}_{\tau}^{y} ; \widehat{\boldsymbol{\mu}}_{\tau c}^{y}, \widehat{\boldsymbol{\Sigma}}_{\tau c}^{y}, \boldsymbol{a}_{y}, \boldsymbol{b}_{y}\right) d \boldsymbol{\theta}_{\tau}^{x} d \boldsymbol{\theta}_{\tau}^{y},
\end{aligned}
$$

where $f_{T}(\cdot)$ is the truncated multivariate normal distribution defined in (8). The RUL is the area under the survival curve (Klein and Moeschberger 2003). Thus, the RUL of the in-service unit $\tau$ predicted at the current time $t_{c}$ can be estimated as:

$$
\widehat{R U L}\left(t \mid t_{c}, \boldsymbol{x}_{\tau, 1: c}, \boldsymbol{y}_{\tau, 1: c}\right)=\int_{t_{c}}^{\infty} S\left(t \mid t_{c}, \boldsymbol{x}_{\tau, 1: c}, \boldsymbol{y}_{\tau, 1: c}\right) d t
$$

where the marginal survival function is given in (17). The overall prognostic framework including offline parameter estimation, online model updating, and RUL prediction is shown in Figure 4.

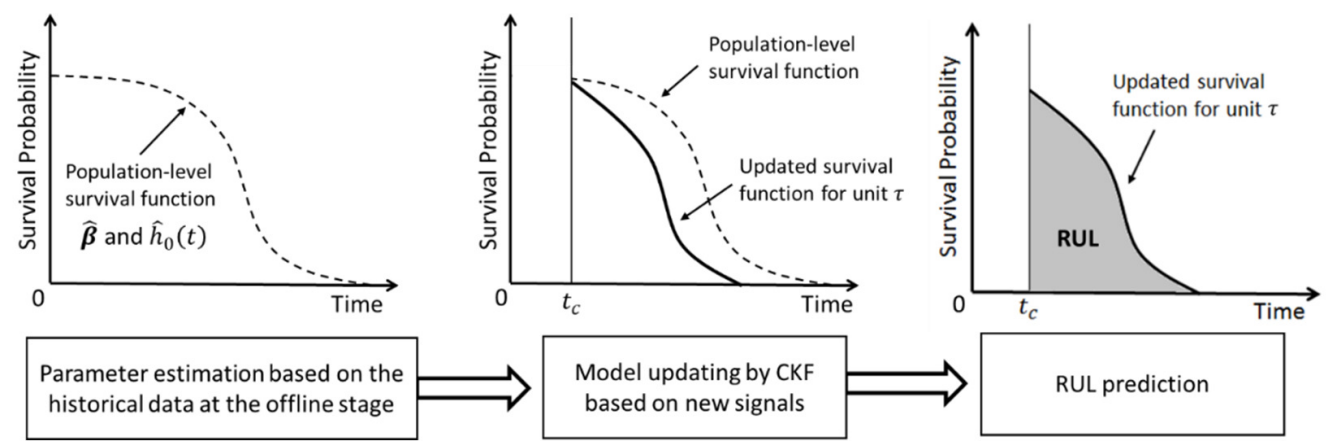

Figure 4. Summary of the overall prognostic framework

\section{Numerical Study}

\subsection{Design of the numerical study}

The first step for the numerical study is to specify the true underlying model and the associated parameters that generate the data. The true model is assumed as

$$
\begin{aligned}
h_{i}(t)=(5.0025 & \left.\times 10^{-8} \times t^{0.0005}\right) \\
& \times \exp \left\{0.05\left[\mathbf{z}_{i}^{x}(t)\right]^{T} \boldsymbol{\theta}_{i}^{x}+20\left[\mathbf{z}_{i}^{y}(t)\right]^{T} \boldsymbol{\theta}_{i}^{y}-0.003\left[\mathbf{z}_{i}^{x}(t)\right]^{T} \boldsymbol{\theta}_{i}^{x}\left[\mathbf{z}_{i}^{y}(t)\right]^{T} \boldsymbol{\theta}_{i}^{y}\right\} .
\end{aligned}
$$


The true model (19) is similar to our model formulation in (15) except for the baseline hazard. The true baseline hazard is assumed to be the Weibull distribution defined as $h_{0}(t)=5.0025 \times$ $10^{-8} \times t^{0.0005}$. The true regression coefficient is specified as $\beta_{0}=0.05, \beta_{1}=20$, and $\beta_{2}=$ -0.003 . Furthermore, the CM signal model parameters $\boldsymbol{\theta}_{i}^{x}$ and $\boldsymbol{\theta}_{i}^{y}$ are assumed to follow truncated multivariate normal distributions $\operatorname{TN}\left(\boldsymbol{\mu}_{x}, \boldsymbol{\Sigma}_{x}, \boldsymbol{a}_{x}, \boldsymbol{b}_{x}\right)$ and $\operatorname{TN}\left(\boldsymbol{\mu}_{y}, \boldsymbol{\Sigma}_{y}, \boldsymbol{a}_{y}, \boldsymbol{b}_{y}\right)$ with parameter specifications given in Table 1 . Lastly, $\boldsymbol{z}_{i}^{x}(t)$ and $\boldsymbol{z}_{i}^{y}(t)$ are specified as a quadratic function of time, i.e., $\boldsymbol{z}_{i}^{x}(t)=\boldsymbol{z}_{i}^{y}(t)=\left[1 t t^{2}\right]^{T}$. The true model and parameter specifications are determined in the way that is similar to the real signal evolution paths shown later in the case study. The random noises for $x$ and $y$ signals are assumed to follow $\varepsilon_{x} \sim N\left(0, \sigma_{x}^{2}\right)$ and $\varepsilon_{y} \sim N\left(0, \sigma_{y}^{2}\right)$, respectively. The true parameters presented in Table 1 are only used to generate the data. Thus, all of the parameters have to be estimated based on the simulated data.

Table 1. True parameter specification for two CM signals

\begin{tabular}{|c|c|c|c|c|c|c|c|}
\hline \multicolumn{4}{|c|}{ CM signal $x$} & \multicolumn{4}{|c|}{ CM signal $y$} \\
\hline $\boldsymbol{\mu}_{x}$ & \multicolumn{3}{|c|}{$\left.\begin{array}{llll}23 & 0.2 & 0.001\end{array}\right]^{T}$} & $\boldsymbol{\mu}_{y}$ & \multicolumn{3}{|c|}{$\left[\begin{array}{llll}0.35 & 0.002 & 0.00001\end{array}\right]^{T}$} \\
\hline $\boldsymbol{\Sigma}_{x}$ & {$\left[\begin{array}{c}4106.6704 \\
96.4692 \\
0.4090\end{array}\right.$} & $\begin{array}{c}96.4692 \\
3.0567 \\
0.0133\end{array}$ & $\left.\begin{array}{c}0.4090 \\
0.0133 \\
0.002\end{array}\right]$ & $\boldsymbol{\Sigma}_{y}$ & {$\left[\begin{array}{c}1.5 \\
0.02 \\
0.000002\end{array}\right.$} & $\begin{array}{c}0.02 \\
0.0005 \\
0.0001\end{array}$ & $\left.\begin{array}{c}0.0001 \\
0.000002 \\
0.0005\end{array}\right]$ \\
\hline $\boldsymbol{a}_{x} \leq \boldsymbol{\theta}_{i}^{x} \leq \boldsymbol{b}_{x}$ & \multicolumn{3}{|c|}{$\begin{array}{c}0 \leq \theta_{i, 1}^{x} \leq 150, \\
0.05 \leq \theta_{i, 2}^{x} \leq 0.2, \text { and } \\
0.00002 \leq \theta_{i, 3}^{x} \leq 0.00004\end{array}$} & $\boldsymbol{a}_{y} \leq \boldsymbol{\theta}_{i}^{y} \leq \boldsymbol{b}_{y}$ & \multicolumn{3}{|c|}{$\begin{array}{c}0 \leq \theta_{i, 1}^{y} \leq 0.7 \\
0.0005 \leq \theta_{i, 2}^{y} \leq 0.001, \text { and } \\
0.000002 \leq \theta_{i, 3}^{y} \leq 0.000004\end{array}$} \\
\hline
\end{tabular}

We assume that a set of records with size of $N$ is simulated prior to the RUL prediction. The data corresponding to each unit $i$ can be denoted as $D_{i}=\left\{V_{i}, \delta_{i}, \boldsymbol{x}_{i}^{h}, \boldsymbol{y}_{i}^{h}\right\}$ where $V_{i}=\min \left(T_{i}, C_{i}\right)$ is the event time. Each unit can either fail at $T_{i}$ or be censored at $C_{i}$ without knowing $T_{i}$. The corresponding event indicator is defined as $\delta_{i}=1$ or 0 indicating whether the $i$ th unit is failed or censored. The histories of the two CM signals are $\boldsymbol{x}_{i}^{h}=\left[x_{i 1}, \ldots, x_{i n_{i}}\right]^{T}$ and $\boldsymbol{y}_{i}^{h}=\left[y_{i 1}, \ldots, y_{i n_{i}}\right]^{T}$ respectively, where $n_{i}$ is the number of $\mathrm{CM}$ signal observations for the $i$ th unit. With this historical data, we first estimate the necessary parameters in the offline stage and then move on to the online model updating and RUL prediction for a specific unit of our interest, say unit $\tau$. In the offline stage, following the two-stage estimation procedure, we first need to compute the fitted CM signal values $\hat{x}_{i}(t)=\left[\boldsymbol{z}_{i}^{x}(t)\right]^{T} \widehat{\boldsymbol{\mu}}_{i n_{i}}^{x *}$ and $\hat{y}_{i}(t)=\left[\boldsymbol{z}_{i}^{y}(t)\right]^{T} \hat{\boldsymbol{\mu}}_{i n_{i}}^{y *}$ for each and every unit $i$ (the first stage). In other words, we run the CKF based on the entire CM signal histories $\boldsymbol{x}_{i}^{h}$ and $\boldsymbol{y}_{i}^{h}$ to get $\widehat{\boldsymbol{x}}_{i}^{h}$ and 
$\widehat{\boldsymbol{y}}_{i}^{h}$. Then, the second stage is to estimate $\boldsymbol{\beta}$ based on $\widehat{\boldsymbol{x}}_{i}^{h}$ and $\widehat{\boldsymbol{y}}_{i}^{h}$ by using the maximum likelihood method (Klein and Moeschberger 2003).

In this numerical study, we have specified the sample size of the historical data $N$ as 100 . We have evaluated the prediction accuracy by mean absolute error (MAE) that is defined as

$$
M A E=\frac{1}{M} \sum_{\tau=1}^{M}\left|R U L_{\tau}\left(t \mid t_{c} ; \boldsymbol{\theta}_{\tau}^{x}, \boldsymbol{\theta}_{\tau}^{y}, h_{0}(t), \boldsymbol{\beta}\right)-\widehat{R U L}_{\tau}\left(t \mid t_{c}, \boldsymbol{x}_{\tau, 1: c}, \boldsymbol{y}_{\tau, 1: c}, \hat{h}_{0}(t), \widehat{\boldsymbol{\beta}}\right)\right|
$$

where $\widehat{R U L}_{\tau}$ is the predicted RUL of unit $\tau$ at time $t_{c}$ defined in (18) and $R U L_{\tau}$ is the true RUL of unit $\tau$ computed based on the true parameters $\boldsymbol{\theta}_{\tau}^{x}, \boldsymbol{\theta}_{\tau}^{y}, h_{0}(t)$, and $\boldsymbol{\beta}$. The MAE is computed by averaging over $M$ iterations. The overall simulation procedure is summarized in Table 2.

Table 2. Summary of the overall simulation procedures

\section{Simulate data and testing case}

Step 1: Generate realization of $\boldsymbol{\theta}_{i}^{x}$ and $\boldsymbol{\theta}_{i}^{y}$ for each of the $N=100$ units based on Table 1.

Step 2: Generate the failure time $T_{i}$ by drawing a random sample from the failure time distribution $f_{i}(t)=h_{i}(t) S_{i}(t)$ where the hazard $h_{i}(t)$ is defined in (19) and the survival function is defined as $S_{i}(t)=\exp \left[-\int_{0}^{t} h_{i}(u) d u\right]$.

Step 3: Randomly select $5 \%$ of the units $(0.05 \times N)$ and censor their failure times $T_{i} \rightarrow C_{i}$. The censored time $C_{i}$ is generated from uniform distribution $\mathrm{U}\left(0, T_{i}\right)$.

Step 4: Generate two CM signals based on $\boldsymbol{\theta}_{i}^{x}$ and $\boldsymbol{\theta}_{i}^{y}$ for $N$ units until their end time $V_{i}=$ $\min \left(T_{i}, C_{i}\right)$ with random noise $\varepsilon_{x} \sim N\left(0, \sigma_{x}^{2}\right)$ and $\varepsilon_{y} \sim N\left(0, \sigma_{y}^{2}\right)$. More specifically, the signals are generated by $x_{i}(t)=\left[1 t t^{2}\right] \boldsymbol{\theta}_{i}^{x}+\varepsilon_{x}(t)$ and $y_{i}(t)=\left[1 t t^{2}\right] \boldsymbol{\theta}_{i}^{y}+\varepsilon_{y}(t)$.

Step 5: Generate realization of $\boldsymbol{\theta}_{\tau}^{x}$ and $\boldsymbol{\theta}_{\tau}^{y}$ for unit $\tau$ and generate realizations of the two CM signals $\boldsymbol{x}_{\tau, 1: 999}$ and $\boldsymbol{y}_{\tau, 1: 999}$ (testing unit).

\section{Offline: Parameter estimation}

Step 6: The CKF is run for each of the $N$ units to obtain $\widehat{\boldsymbol{x}}_{i}^{h}$ and $\widehat{\boldsymbol{y}}_{i}^{h}$ (the first stage of two-stage estimation method).

Step 7: Using the fitted signals $\widehat{\boldsymbol{x}}_{i}^{h}$ and $\widehat{\boldsymbol{y}}_{i}^{h}$, we estimate $\boldsymbol{\beta}$ (the second stage of two-stage estimation method) and the baseline hazard $h_{0}(t)$ is estimated by the smooth spline (non-parametrically).

Online: Model updating and RUL prediction for unit $\tau$ (depends on the current time instant)

Step 8: Specify the current time instant $t_{c}$ where $t_{c}<999$.

Step 9: Run the CKF on $\boldsymbol{x}_{\tau, 1: c}$ and $\boldsymbol{y}_{\tau, 1: c}$ to get $\widehat{\boldsymbol{\mu}}_{\tau c}^{x *}, \widehat{\boldsymbol{\Sigma}}_{\tau c}^{x *}, \widehat{\boldsymbol{\mu}}_{\tau c}^{y *}$, and $\widehat{\boldsymbol{\Sigma}}_{\tau c}^{y *}$.

Step 10: Perform RUL prediction based on (18) and compute the absolute prediction error.

Step 11: Increase the current time instant $t_{c}$ and repeat Steps 8-10.

Step 12: Repeat Steps 1-11 for $M$ iterations to compute the mean absolute error defined in (20). 
To run the CKF in Step 6 and Step 9, we need to initialize the filter. In other words, the initial guess for parameters $\widehat{\boldsymbol{\mu}}_{0}^{x}, \widehat{\boldsymbol{\Sigma}}_{0}^{x}, \widehat{\boldsymbol{\mu}}_{0}^{y}$, and $\widehat{\boldsymbol{\Sigma}}_{0}^{y}$ is required for both CM signals. It is generally agreed that there is no universally-accepted rule for initializing stochastic filters. In many cases, the initialization of the filter is done by manual tuning guided by some domain knowledge (Simon and Simon 2010; Lim and Mba 2015). The filter initialization has not been emphasized because the impact of initialization gradually reduces as the observations accumulate. Specifying the initial parameters $\widehat{\boldsymbol{\mu}}_{0}^{x}, \widehat{\boldsymbol{\Sigma}}_{0}^{x}, \widehat{\boldsymbol{\mu}}_{0}^{y}$, and $\widehat{\boldsymbol{\Sigma}}_{0}^{y}$ is equivalent to specifying priors in the conventional Bayesian model updating framework, which typically depends on the availability of the relevant historical data and/or domain knowledge acquired by experts (Zhou et al. 2014). Therefore, in our case, it is natural to initialize the CKF based on the historical data. More specifically, we fit the mixed effects model defined in (1) to each of the signals $\boldsymbol{x}_{i}^{h}$ and $\boldsymbol{y}_{i}^{h}$ for all $N$ units to estimate $\widehat{\boldsymbol{\mu}}_{x}$ and $\widehat{\boldsymbol{\Sigma}}_{x}$ as well as $\widehat{\boldsymbol{\mu}}_{y}$ and $\widehat{\boldsymbol{\Sigma}}_{y}$. This can be done by restricted maximum likelihood approach (Laird and Ware 1982). Then, we use those offline estimates to initialize the filters, i.e., $\widehat{\boldsymbol{\mu}}_{0}^{x}=\widehat{\boldsymbol{\mu}}_{x}, \widehat{\boldsymbol{\Sigma}}_{0}^{x}=\widehat{\boldsymbol{\Sigma}}_{x}, \widehat{\boldsymbol{\mu}}_{0}^{y}=\widehat{\boldsymbol{\mu}}_{y}$, and $\widehat{\boldsymbol{\Sigma}}_{0}^{y}=\widehat{\boldsymbol{\Sigma}}_{y}$. Also, the measurement noise for signals $\sigma_{x}^{2}$ and $\sigma_{y}^{2}$ can be estimated in this way.

\subsection{Performance evaluation}

We generate the CM signals with different noise levels to see the impact of noise level on the RUL prediction accuracy. Figure 5 shows the simulated $x$ and $y$ signals under various noise levels where solid lines represent the true CM signal path.
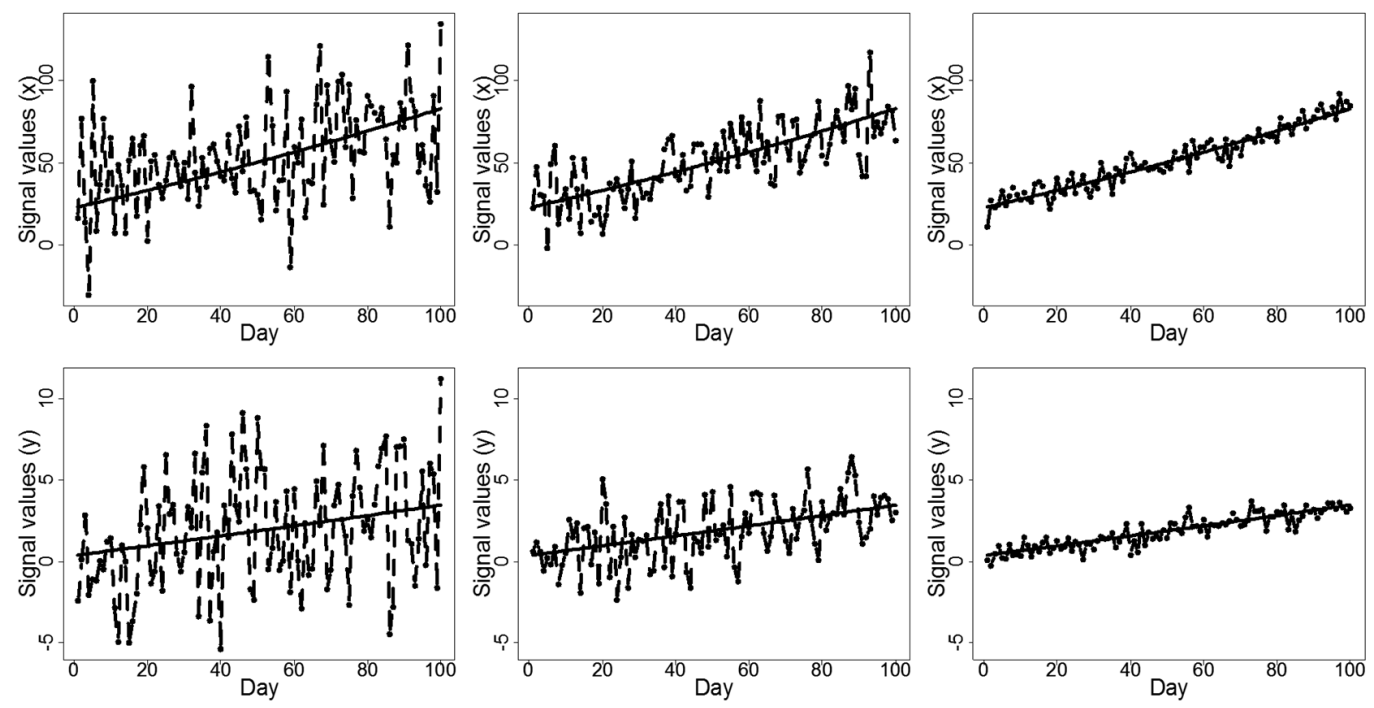

(a) $\sigma_{x}=40$ and $\sigma_{y}=3$

(b) $\sigma_{x}=10$ and $\sigma_{y}=1$

(c) $\sigma_{x}=5$ and $\sigma_{y}=0.5$

Figure 5. Simulated CM signals with different noise levels 
From Figure 5, we can imagine that, under case $(a)$, prediction could fail often if we use the conventional Bayesian method (denoted as BM) without enforcing constraints. On the other hand, under case (c), both CKF and BM would work satisfactorily because of the moderate noise level.

To show the advantageous features of the CKF, we present an illustration in Figure 6 using an example unit under case (a). In Figure 6(a), at $t_{c}=50$, although the estimated survival curve based on the CKF is more accurate than the one based on the BM, we can say both CKF and BM perform acceptably. However, when we have more signal observations up to $t_{c}=250$, the CM signal model for signal $x$ updated by the BM shows a decreasing trend as shown in Figure 6(b). This falsely estimated signal path inevitably yields overestimated survival function and inflates RUL prediction. On the other hand, the CKF method captures the true survival curve quite accurately at $t_{c}=250$. The monotonicity, i.e., $\mathbf{0} \leq \boldsymbol{\theta}_{i}^{x}$ and $\mathbf{0} \leq \boldsymbol{\theta}_{i}^{y}$ (non-negative coefficients), is always a reasonable constraint because the underlying health status of any in-situ units cannot get better unless some maintenance actions were performed. Thus, by simply restricting the CM signal model parameters to be strictly non-negative, we can improve the prediction accuracy significantly.

(a)
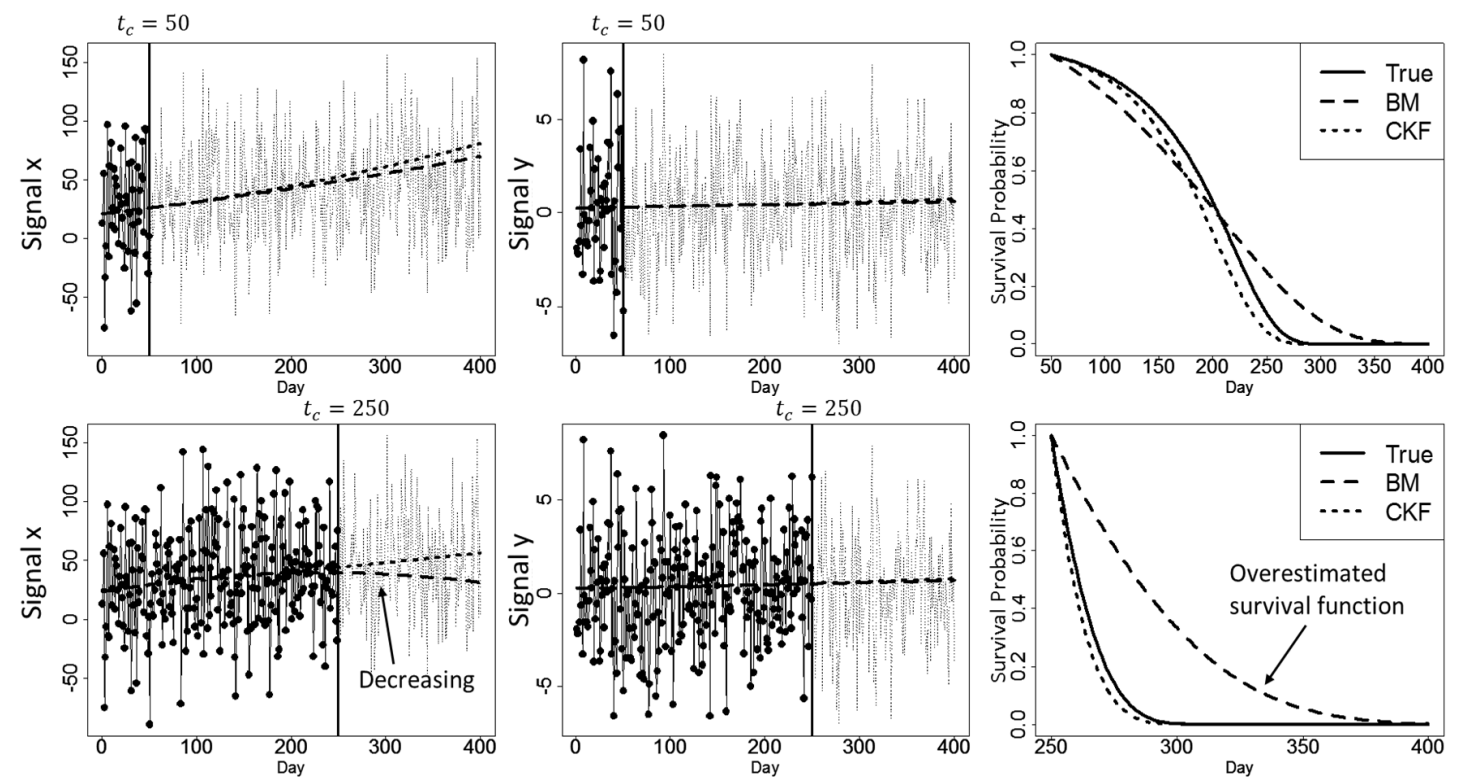

Figure 6. Illustration of the benefit of the proposed CKF method under case (a): $\sigma_{x}=40$ and $\sigma_{y}=3$ where the constraints for the CKF are $\mathbf{0} \leq \boldsymbol{\theta}_{i}^{x}$ and $\mathbf{0} \leq \boldsymbol{\theta}_{i}^{y}$ (non-negativity)

As we can expect, the performance of the BM highly depends on the level of random noise. When the observed CM signals are contaminated by large amount of random noise, the BM often fails to capture the true underlying CM signal evolution path as illustrated in Figure 6. However, 
when the noise is negligible, the BM would also perform satisfactorily. Therefore, the prediction accuracy of the BM and proposed CKF methods needs to be compared under various noise levels.

Table 3 reports the performance evaluation and comparison results where the CKF assumes non-negativity constraints, i.e., $\mathbf{0} \leq \boldsymbol{\theta}_{i}^{x}$ and $\mathbf{0} \leq \boldsymbol{\theta}_{i}^{y}$. The prediction failure in Table 3 is defined as the cases where $\widehat{R U L}_{\tau}>5000$ (days). In other words, when the predicted RUL is larger than 5000 days (about 13.7 years), we consider $\widehat{R U L}_{\tau}=$ Infinite. The MAEs for the BM in Table 3 are computed solely based on the feasible RUL predictions, i.e., all infinite RUL predictions were excluded from the MAE computation.

Table 3. Performance evaluation under three different noise levels $(M=5000)$

\begin{tabular}{|c|c|c|c|c|c|c|c|}
\hline \multirow{2}{*}{ Case } & \multirow{2}{*}{ Method } & \multirow{2}{*}{ Criteria } & \multicolumn{5}{|c|}{ Prediction time instant $t_{c}$} \\
\hline & & & 10 & 50 & 150 & 250 & 300 \\
\hline \multirow{3}{*}{ (a) } & CKF & $\begin{array}{c}\text { MAE } \\
\text { (std error) }\end{array}$ & $\begin{array}{c}92.145 \\
(60.760)\end{array}$ & $\begin{array}{c}79.779 \\
(49.812)\end{array}$ & $\begin{array}{c}51.955 \\
(34.559)\end{array}$ & $\begin{array}{c}29.322 \\
(26.462)\end{array}$ & $\begin{array}{c}19.890 \\
(25.366)\end{array}$ \\
\hline & \multirow{2}{*}{$\mathrm{BM}$} & $\begin{array}{c}\text { MAE } \\
\text { (std error) }\end{array}$ & $\begin{array}{c}82.600 \\
(51.793)\end{array}$ & $\begin{array}{c}75.799 \\
(50.097)\end{array}$ & $\begin{array}{c}73.229 \\
(66.922)\end{array}$ & $\begin{array}{c}64.954 \\
(112.178)\end{array}$ & $\begin{array}{c}54.579 \\
(115.251)\end{array}$ \\
\hline & & $\begin{array}{c}\text { Prediction } \\
\text { failures }(\%)\end{array}$ & 17.761 & 18.049 & 21.763 & 19.459 & 16.077 \\
\hline \multirow{3}{*}{ (b) } & CKF & $\begin{array}{c}\text { MAE } \\
\text { (std error) }\end{array}$ & $\begin{array}{c}74.740 \\
(47.062)\end{array}$ & $\begin{array}{c}48.979 \\
(32.042)\end{array}$ & $\begin{array}{c}22.115 \\
(18.515)\end{array}$ & $\begin{array}{c}7.957 \\
(10.637)\end{array}$ & $\begin{array}{c}3.797 \\
(7.023)\end{array}$ \\
\hline & \multirow{2}{*}{$\mathrm{BM}$} & $\begin{array}{c}\text { MAE } \\
\text { (std error) }\end{array}$ & $\begin{array}{c}70.368 \\
(45.506)\end{array}$ & $\begin{array}{c}50.090 \\
(35.596)\end{array}$ & $\begin{array}{c}33.988 \\
(36.125)\end{array}$ & $\begin{array}{c}21.711 \\
(59.595)\end{array}$ & $\begin{array}{c}12.395 \\
(47.299)\end{array}$ \\
\hline & & $\begin{array}{c}\text { Prediction } \\
\text { failures }(\%)\end{array}$ & 7.120 & 7.554 & 7.610 & 6.526 & 3.431 \\
\hline \multirow{3}{*}{ (c) } & $\mathrm{CKF}$ & $\begin{array}{c}\text { MAE } \\
\text { (std error) }\end{array}$ & $\begin{array}{c}59.446 \\
(36.661)\end{array}$ & $\begin{array}{c}28.729 \\
(20.071)\end{array}$ & $\begin{array}{c}11.952 \\
(12.841)\end{array}$ & $\begin{array}{c}4.947 \\
(8.171)\end{array}$ & $\begin{array}{c}2.264 \\
(5.340)\end{array}$ \\
\hline & \multirow{2}{*}{$\mathrm{BM}$} & $\begin{array}{c}\text { MAE } \\
\text { (std error) }\end{array}$ & $\begin{array}{c}57.698 \\
(37.662)\end{array}$ & $\begin{array}{c}30.435 \\
(21.022)\end{array}$ & $\begin{array}{c}13.654 \\
(14.051)\end{array}$ & $\begin{array}{c}6.581 \\
(9.156)\end{array}$ & $\begin{array}{c}3.557 \\
(6.291)\end{array}$ \\
\hline & & $\begin{array}{c}\text { Prediction } \\
\text { failures }(\%)\end{array}$ & 0.989 & 1.014 & 1.112 & 0.782 & 0.528 \\
\hline
\end{tabular}

From Table 3, we can make following observations. First, the prediction accuracies for both BM and CKF improve as prediction time instant $t_{c}$ increases and as the random noise level decreases which is intuitively understandable. Second, even though the infinite RUL predictions were excluded, the MAEs of the BM are still larger than the CKF. This phenomenon is due to the inflated 
RUL prediction illustrated in Figure 6 and explicitly shows the better performance of the CKFbased prognosis. However, as expected, when the signal has small amount of noise, i.e., case (c), the BM performs very similar to the proposed CKF and the prediction failures only occur, on average, 0.88 percent. Thus, if CM signals do not involve high level of noise, the proposed CKF method may not be necessary. On the other hand, with a high noise level, the BM often fails to provide reasonable RUL predictions. For instance, under the case $(a)$ where $\sigma_{x}=40$ and $\sigma_{y}=3$, the JPM based on the BM fails to give realistic RUL predictions about $16 \%$ to $21 \%$ of times whereas the JPM based on the CKF never experiences such an issue.

\subsection{Discussion on the performance of the $\mathrm{CKF}$ with additional constraints}

In the previous section, we only have enforced single-sided constraints, i.e., all parameters are non-negative. However, we could have more precise constraints based on the physical knowledge of the degradation process. The proposed CKF is flexible to accommodate the double-sided inequality constraints. Thus, in this section, we shall see the improvement of having more precise constraints. Now, we specify the constraints for the signal $x$ as

$$
0 \leq \theta_{i, 1}^{x} \leq 150,0 \leq \theta_{i, 2}^{x} \leq 0.2 \text {, and } 0 \leq \theta_{i, 3}^{x} \leq \mathrm{Inf},
$$

and the constraints for the signal $y$ are specified as

$$
0 \leq \theta_{i, 1}^{y} \leq 0.7,0 \leq \theta_{i, 2}^{y} \leq 0.001 \text {, and } 0 \leq \theta_{i, 3}^{y} \leq \operatorname{Inf} .
$$

Basically, we assume that the proper upper bounds for the intercept and slope can be defined in addition to the non-negativity constraints. Table 4 shows the MAE and standard errors for CKF method with and without additional constraints under case (a).

Table 4. Performance evaluation with additional constraints under case (a) (M=5000)

\begin{tabular}{ccccccc}
\hline \multirow{2}{*}{ Method } & \multirow{2}{*}{ Criteria } & \multicolumn{5}{c}{ Prediction time instant $t_{c}$} \\
\cline { 3 - 7 } & & 10 & 50 & 150 & 250 & 300 \\
\hline \hline CKF & MAE & 92.145 & 79.779 & 51.955 & 29.322 & 19.890 \\
(lower bound) & (std error) & $(60.760)$ & $(49.812)$ & $(34.559)$ & $(26.462)$ & $(25.366)$ \\
\hline CKF & MAE & 85.411 & 71.905 & 44.809 & 27.818 & 18.892 \\
(lower and upper bound) & (std error) & $(56.449)$ & $(47.705)$ & $(31.141)$ & $(25.990)$ & $(23.847)$ \\
\hline
\end{tabular}

From Table 4, we can clearly see the improvement introduced by having additional constraints. The reward of having additional constraints gradually diminishes as $t_{c}$ increases. However, regardless of $t_{c}$, the RUL prediction with additional constraints is always more accurate than the one without upper bounds of the inequality constraints. However, as noted before, we can easily foresee that the proposed CKF would perform unsatisfactorily with falsely specified constraints. 
Therefore, if investigators are not certain about the nature of the degradation process, the tight constraints should not be enforced.

\section{Case study}

To validate the advantageous features of the proposed CKF method, we perform a case study with real world data in the application of automotive lead-acid battery. The lead-acid battery is a crucial component that ignites the engine of the automotive vehicle. When the battery fails to perform its functionality, the engine cannot be started. Thus, the battery needs to be either repaired or replaced in advance to the failure so that such catastrophic consequences can be avoided.

\subsection{Description of the RUL prognosis for automotive lead-acid batteries}

The data contains two CM signals that are known to be strongly associated with the battery health status. Due to the confidentiality issue, we denote those two CM signals as signal $x$ and $y$ similar to the notation in numerical study. For the same issue, the time is masked to conceal the actual failure times of the batteries.

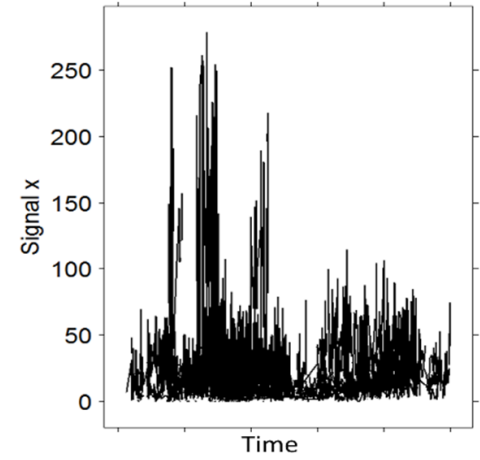

(a) Signal observations for $x$ and $y$

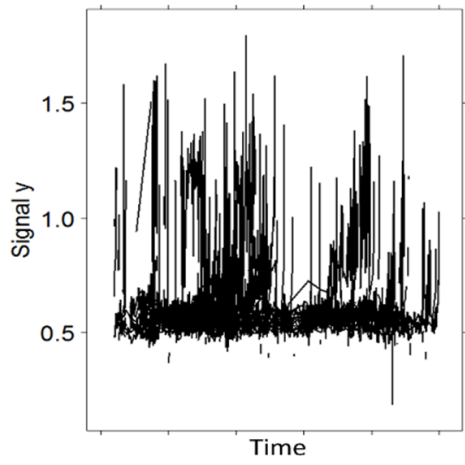

Figure 7. CM signals and failure time data for 50 automotive lead-acid batteries

From Figure 7(a), we can see that both signals exhibit a high level of noise with many abrupt ups and downs. Thus, the conventional BM may not be effective for the online prognosis. In total, 50 batteries are monitored until failure where failure time is defined as the time when the batteries are replaced. Figure 7(b) shows the histogram of the failure times for 50 automotive lead-acid batteries. From Figure 7(b), we can see that the failure time distribution shows a large variance where some of the batteries have died early whereas others have lasted for longer duration.

\subsection{Illustration of the CKF-based RUL prognostic method}

Before moving on to the performance evaluation, we show some practical examples where the prognostic method based on the conventional BM fails to provide reasonable RUL prediction. 
Figure 8(a) and 8(b) show the $x$ and $y$ signals collected from two example batteries $\mathrm{A}$ and $\mathrm{B}$, respectively, where the prediction time instants $t_{c, A}$ and $t_{c, B}$ for the batteries $\mathrm{A}$ and $\mathrm{B}$ are shown by vertical solid lines. The updated signal models based on CKF and BM are shown in addition to the actual signal observations. Also, survival curves that correspond to each CKF and BM are shown. From the estimated survival curve, the RUL is predicted and the MAEs are reported. The constraints for the CKF is specified as $\mathbf{0} \leq \boldsymbol{\theta}_{i}^{x}$ and $\mathbf{0} \leq \boldsymbol{\theta}_{i}^{y}$, i.e., non-negativity constraints.

(a)
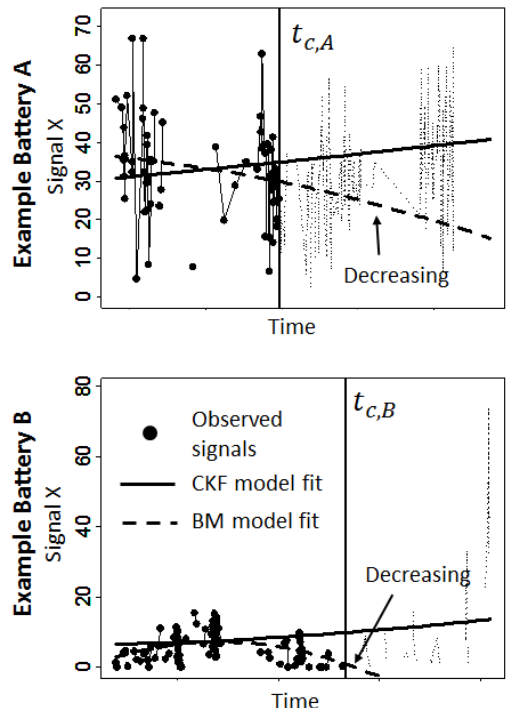
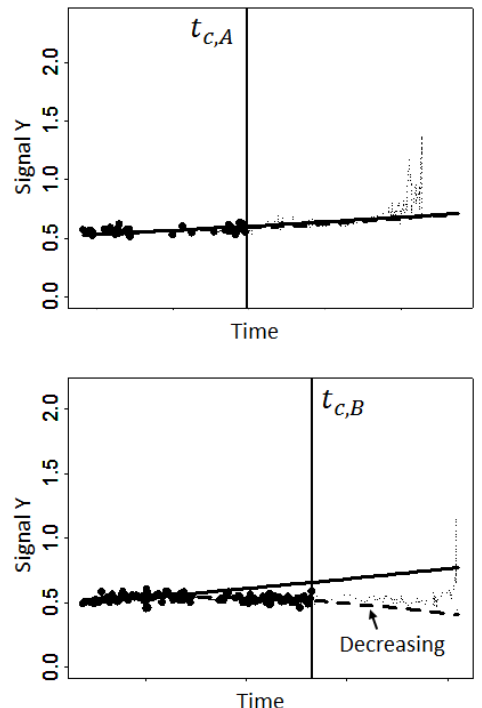
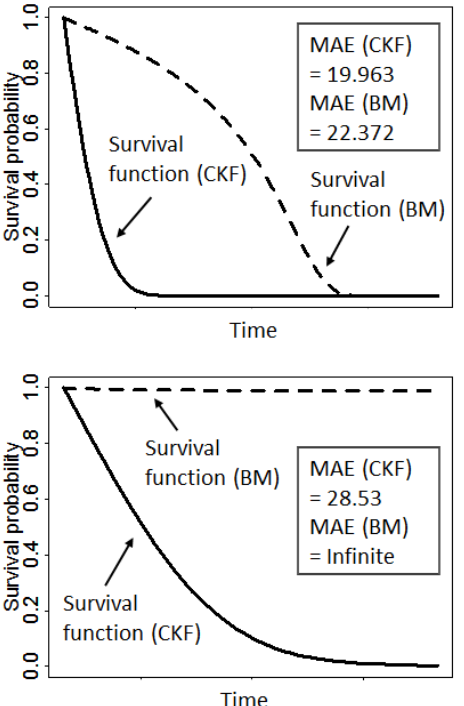

Figure 8. Illustration of the advantageous features of the proposed CKF method with real data

Based on Figure 8, we can observe the following. First, for battery A, the BM captures an unreasonable decreasing trend in signal $x$ at $t_{c, A}$ resulting an inflated survival function. Due to this inflation in the survival probability, the BM-based JPM overestimates the RUL compared to the CKF-based JPM as shown in Figure 8(a). For battery B, the situation gets even worse for BM. The updated signal model based on the BM shows a decreasing trend in both signals $x$ and $y$ at $t_{c, B}$ which yields very flat survival curve around 1. Because the estimated survival probability minimally decreases over time, the predicted RUL becomes almost infinite. On the other hand, the CKF-based JPM provides acceptable RUL prediction as illustrated in Figure 8(b). Therefore, based on Figure 8, we can see the practical advantages of the proposed CKF method.

\subsection{Performance evaluation}

To perform a comprehensive performance evaluation, we use leave-one-out cross-validation approach. We first exclude one battery at the very beginning and use the rest of the batteries $(N=49)$ for offline model fitting. Based on the estimated parameters using 49 batteries, the online RUL 
prediction for a specific battery that was excluded at the beginning is performed. The whole procedure is repeated 50 times, i.e., $M=50$, to compute the MAE defined in (20). The prediction time instances $t_{c}$ are defined as $\omega$-percentile, i.e., $t_{c, i}=\omega T_{i}$. In other words, each battery has different prediction time instances $t_{c, i}$ defined by the specific percentile $\omega$ and its failure time $T_{i}$. The percentiles $\omega$ are specified as $20 \%, 30 \%, 50 \%, 70 \%$, and $95 \%$. For instance, if a battery is failed at time 100, we perform the online RUL prediction at 20,30, 50, 70, and 95. The CKF has non-negative constraints as in the numerical study. Table 5 summarizes the results.

Table 5. Performance evaluation based on real world data with non-negativity constraints

\begin{tabular}{ccccccc}
\hline \multirow{2}{*}{ Method } & \multirow{2}{*}{ Criteria } & \multicolumn{5}{c}{ Prediction percentile $\omega$} \\
\cline { 3 - 7 } & & $20 \%$ & $30 \%$ & $50 \%$ & $70 \%$ & $95 \%$ \\
\hline \hline \multirow{2}{*}{ CKF } & MAE & 100.897 & 75.727 & 47.721 & 33.172 & 13.514 \\
& (std. error) & $(93.921)$ & $(75.323)$ & $(50.065)$ & $(30.436)$ & $(17.430)$ \\
\hline \multirow{3}{*}{ BM } & $\begin{array}{c}\text { MAE } \\
\text { (std. error) }\end{array}$ & $\begin{array}{c}96.117 \\
(80.165)\end{array}$ & $\begin{array}{c}81.224 \\
(94.635)\end{array}$ & $\begin{array}{c}66.223 \\
(51.145)\end{array}$ & $\begin{array}{c}74.140 \\
(78.117)\end{array}$ & $\begin{array}{c}50.940 \\
(46.982)\end{array}$ \\
\cline { 2 - 7 } & $\begin{array}{c}\text { Prediction } \\
\text { failures (\%) }\end{array}$ & $6 \%$ & $4 \%$ & $6 \%$ & $8 \%$ & $6 \%$ \\
\hline
\end{tabular}

Similar to the numerical study, the infinite RUL predictions are excluded from the MAE computation for the BM. From Table 5, we can see that the CKF-based JPM performs significantly better except for the very early stage of prediction at 20-percentile. The MAE of CKF monotonically decreases as we collect more data from the battery (as $\omega$ increases). However, the decreasing trend in MAE for the BM-based JPM is somewhat not as evident as the CKF-based JPM. In other words, having more CM signal data may not be always helpful for the conventional $\mathrm{BM}$ due to the high level of random noise. Even though the infinite RUL predictions were excluded from the MAE computation, in some cases, newly collected signals create the issue of inflated survival function as illustrated in Figure 8(a), i.e., example battery A, which gives unreasonably overestimated RUL prediction. Lastly, as we expected, the BM is not guaranteed to provide a finite RUL prediction all the time. With this case study data, the BM fails to give the finite RUL prediction, on average, $6 \%$ of the time, i.e., fails to provide finite RUL prediction for 2 to 4 batteries among 50 batteries in total.

Figure 9 shows the histogram of RULs for 50 batteries at 50-, 70-, and 95-percentiles. The predicted failure time density curves, i.e., smoothed histogram, for both CKF and BM are plotted as well. It should be noted that the failure densities shown in Figure 9 are not for individual units. 
Figure 9 graphically summarizes the overall prediction accuracy of the RUL prediction based on the cross validation with 50 distinctive predictions performed on the case study data. As we can see from Figure 9, the conventional BM tends to overestimate the failure time whereas the CKFbased prediction satisfactorily predicts the RUL. Therefore, based on both Table 5 and Figure 9, we can conclude that the CKF-based JPM robustly predicts the RUL despite of the high level of random noise. Overall, the case study analysis results based on the real world data performed in this section validates our findings from the numerical study and shows the practical advantages of the RUL prognostic method based on the proposed CKF.
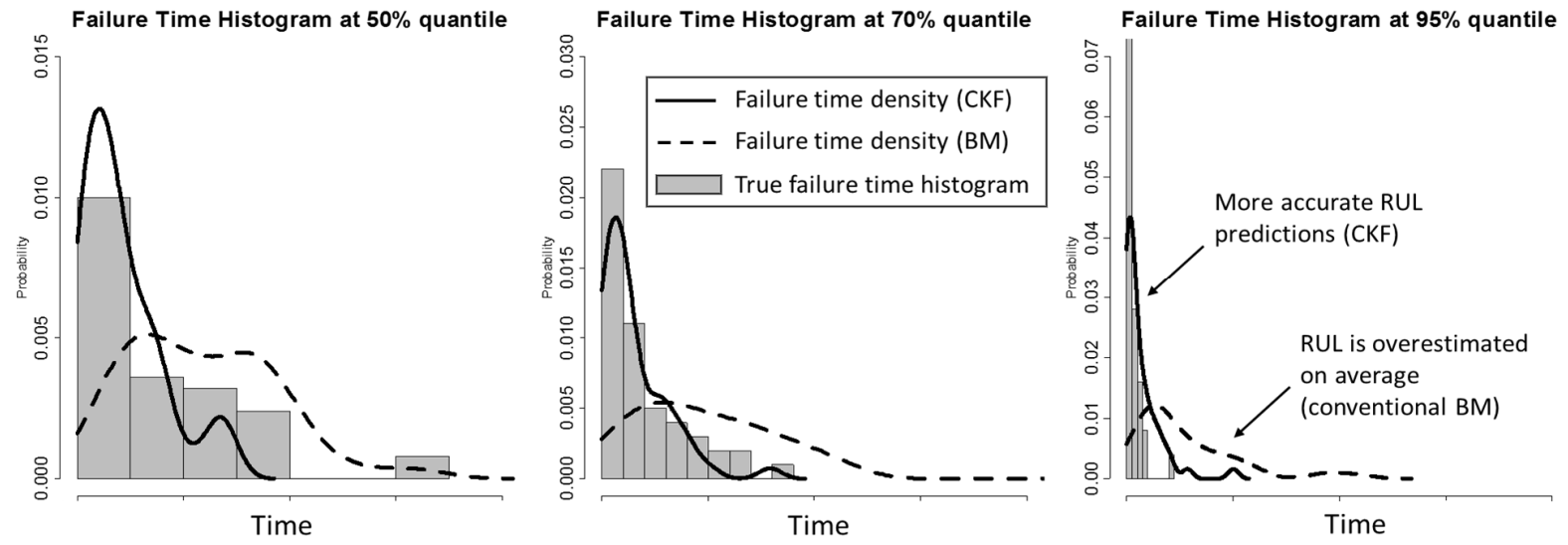

Figure 9. Histogram of the true failure times and predicted failure times where infinite RUL predictions were excluded for plotting the density curves for the BM-based JPM

\section{Conclusion}

In this paper, we proposed a constrained Kalman filtering (CKF) approach to model the $\mathrm{CM}$ signal propagation path. The conventional Bayesian method (BM) often fails when the CM signals are contaminated by a significant amount of noise. On the other hand, the proposed CKF method performs well even under a high level of random noise. The CKF method enforces a set of inequality constraints on the parameter space so that the updated model parameters can stay in the specified region which can be justified by either physical theory of system degradation or empirical domain knowledge from experts. By having those additional information (constraints), the conventional Bayesian model updating method can be significantly improved.

The specific contributions of the proposed prognostic framework in this paper are as follows: first, the CKF method for modeling and updating the CM signal evolution is integrated within the online RUL prognostic framework. In this paper, the joint prognostic model (JPM) is used as an example prognostic method and we have shown that the integrating JPM and CKF can improve 
the prediction accuracy. It needs to be emphasized that the proposed CKF method for modeling the $\mathrm{CM}$ signal evolution is not limited to a specific prognostic algorithm. It can be integrated into any prognostic methods that utilize the online model updating. Second, unlike the existing PDF truncation-based CKF methods based on either sequential constraints enforcement or Monte Carlo sampling techniques, the proposed CKF directly uses the MGF to compute the constrained mean and variance-covariance. By doing so, we can achieve efficient updating for the CM signals.

In this paper, we have considered only two CM signals and their interaction. However, most of the contemporary real time monitoring systems tend to collect several CM signals, typically, more than two. In such case, a sophisticated CM signal selection procedure may need to be performed prior to the model fitting and online prognosis. It is also possible to consider an effective composite health index that combines a subset of multiple CM signals so that the RUL prediction can be conducted based on the user-defined composite health index. Furthermore, in many applications, the CM signal evolution is affected by time-varying environmental and external factors, i.e., RUL of an automotive battery depends on temperature or vehicle usage pattern of individual users. Thus, advanced methods to incorporate such uncontrollable factors are desired to improve the modeling capability. We shall investigate along these lines and report the results in the future.

\section{Acknowledgement}

The financial support for this work was provided by NSF grant CMMI-1335129.

\section{Reference}

Bluvband, Z., Porotsky, S., and Tropper, S., "Critical zone recognition: Classification vs. regression," IEEE Conference on Prognostics and Health Management, 1-5, 2014

Bycott, P. and Taylor, J., "A comparison of smoothing techniques for CD4 data measured with error in a time-dependent Cox proportional hazards model," Statistics in Medicine, 17(18), 2061-2077, 1998

Byon, E., Ntaimo, L., and Ding, Y., "Optimal maintenance strategies for wind turbine systems under stochastic weather conditions," IEEE Transactions on Reliability, 59(2), 393-404, 2010

Cartinhour, J., "One-dimensional marginal density functions of a truncated multivariate normal density function," Communications in Statistics - Theory and Method, 19, 197-203, 1990 
Chen, C., Vachtsevanos, G., Orchard, M., "Machine remaining useful life prediction: an integrated adaptive neuro-fuzzy and high-order particle filtering approach," Mechanical Systems and Signal Processing, 28, 597-607, 2012

Chen, N., and Tsui, K. L., "Condition monitoring and remaining useful life prediction using degradation signals: Revisited," IIE Transactions, 45(9), 939-952, 2013

Gebraeel, N., Lawley, M., Li, R., and Ryan, J., "Residual-life distribution from component degradation signals: A Bayesian approach," IIE Transactions, 3(4), 543-557, 2005

Gorjian, N., Ma, L., Mittinty, M., Yarlagadda, P., and Sun, Y., "A review on degradation models in reliability analysis," Engineering Asset Lifecycle Management - Proceedings of the 4th World Congress on Engineering Asset Management (WCEAM), 369-384, 2010

Klein, J. P. and Moeschberger, M. L., Survival Analysis - Techniques for Censored and Truncated Data, $2^{\text {nd }}$ ed., Springer, 2003

Laird, N. M. and Ware, J. H., "Random-effects models for longitudinal data," Biometrics, 38(4), 963-974, 1982

Lee, L. F., "On the first and second moments of the truncated multi-normal distribution and a simple estimator," Economics Letters, 3, 165-169, 1979

Leppard, P. and Tallis, G. M., "Algorithm AS 249: Evaluation of the mean and covariance of the truncated multinormal distribution," Applied Statistics, 38, 543-553, 1989

Liao, H., Zhao, W., and Guo, H., "Predicting remaining useful life of an individual unit using proportional hazards model and logistic regression model," Proceedings of the Annual Reliability and Maintainability Symposium, Newport Beach, CA, 2006

Lim, C. K. R. and Mba, D., "Switching Kalman filter for failure prognostics," Mechanical Systems and Signal Processing, 52(53), 426-435, 2015

Lu, C. and Meeker, W., "Using degradation measures to estimate a time-to-failure distribution," Technometrics, 35, 161-174, 1993

Manjunath, B. G. and Wilhelm, S., "Moment calculation for the doubly truncated multivariate normal density," arXiv:1206.5387v1, 2012

Muthen, B., "Moments of the censored and truncated bivariate normal distribution," British Journal of Mathematical and Statistical Psychology, 43, 131-143, 1990

Rhodes, I. B., "A tutorial introduction to estimation and filtering," IEEE Transactions on Automatic Control, AC-16(6), 688-706, 1971

Shimada, N., Shirai, Y., Kuno, Y., and Miura, J., "Hand gesture estimation and model refinement using monocular camera - ambiguity limitation by inequality constraints," The $3^{\text {rd }}$ IEEE International Conference on Automatic Face and Gesture Recognition, 268-273, 1998 
Si, X., Wang, W., Hu, C., and Zhou, D., "Remaining useful life estimation - A review on the statistical data driven approaches," European Journal of Operational Research, 213, 1-14, 2011

Si, X-S., Wang, W., Hu, C-H., Chen, M-Y., and Zhou, D-H., "A Wiener-process-based degradation model with a recursive filter algorithm for remaining useful life estimation," Mechanical Systems and Signal Processing, 35, 219-237, 2013

Simon, D. and Chia, T., "Kalman filtering with state equality constraints," IEEE Transcations on Aerospace and Electronic Systems, 39, 128-136, 2002

Simon, D. and Simon, D. L., "Aircraft turbofan engine health estimation using constrained Kalman filtering," Journal of Engineering for Gas Turbines and Power, 127(2), 323-328, 2005

Simon, D. and Simon, D. L., "Constrained Kalman filtering via density function truncation for turbofan engine health estimation,” International Journal of Systems Science, 41(2), 159-171, 2010

Simon, D., "Kalman filtering with state constraints: a survey of linear and nonlinear algorithms," IET Control Theory \& Applications, 4(8), 1303-1318, 2010

Son, J., Zhang, Y., Sankavaram, C., and Zhou, S., "RUL prediction for individual units based on condition monitoring signals with a change point," IEEE Transactions on Reliability, 64(1), 182-196, 2015

Son, J., Zhou, Q., Zhou, S., Mao, X., and Salman, M., "Evaluation and comparison of mixed effects model based prognosis for hard failure," IEEE Transactions on Reliability, 62(2), 379-394, 2013

Straka, O., Dunik, J., and Simandl, M., "Truncation nonlinear filters for state estimation with nonlinear inequality constraints," Automatica, 48, 273-286, 2012

Sun, J., Li, L., and Xi, L., "Modified two-stage degradation model for dynamic maintenance threshold calculation considering uncertainty," IEEE Transactions on Automation Science and Engineering, 9(1), 209-212, 2012

Tallis, G. M., "The moment generating function of the truncated multinormal distribution," Journal of the Royal Statistical Society, Series B (Methodological), 23(1), 223-229, 1961

Tsiatis, A., DeGruttola, V., and Wulfsohn, M. S., "Modeling the relationship of survival to longitudinal data measured with error: Applications to survival and CD4 counts in patients with AIDS," Journal of the American Statistical Association, 90(429), 27-37, 1995

Wulfsohn, M. S. and Tsiatis, A., "A joint model for survival and longitudinal data measured with error," Biometrics, 53, 330-339, 1997 
Ye, Z. S., and Chen, N., "The inverse Gaussian process as a degradation model," Technometrics, 56(3), 302-311, 2014

Ye, Z., Chen, N., and Tsui, K-L., "A Bayesian approach to condition monitoring with imperfect inspections," Quality and Reliability Engineering International, 31, 513-522, 2015

Yu, I. T. and Fuh, C., "Estimation of time to hard failure distributions using a three-stage method," IEEE Transactions on Reliability, 59(2), 405-412, 2010

Zarchan, P. and Musoff, H., Polynomial Kalman filters, Fundamentals of Kalman filtering; A practical approach, $2^{\text {nd }}$ ed., American Institute of Aeronautics and Astronautics, USA, 2005

Zhou, Q., Son, J., Zhou, S., Mao, X., and Salman, M., "Remaining useful life prediction of individual units subject to hard failure," IIE Transactions, 46(10), 1017-1030, 2014

Zhou, R., Gebraeel, N., and Serban, N., "Degradation modeling and monitoring of truncated degradation signals," IIE Transactions, 44(9), 793-803, 2012

Zio, E. and Peloni, G., "Particle filtering prognostic estimation of the remaining useful life of nonlinear components,” Reliability Engineering and System Safety, 96, 403-409, 2011

\section{Appendix}

For notational simplicity, the subscriptions $i$ and $k$ are omitted in this proof.

Define a $d$-dimensional vector $\boldsymbol{v}$ where $\boldsymbol{v} \in \mathbb{R}^{d}$. Then, the MGF of the doubly-truncated multivariate normal distribution can be derived as (Manjunath and Wilhelm 2012):

$$
M(\boldsymbol{v})=E\left(e^{\boldsymbol{v}^{T} \boldsymbol{\theta}}\right)=\int_{\boldsymbol{a}}^{\boldsymbol{b}} e^{\boldsymbol{v}^{T} \boldsymbol{\theta}} f_{T}(\boldsymbol{\theta} ; \widehat{\boldsymbol{\mu}}, \widehat{\boldsymbol{\Sigma}}, \boldsymbol{a}, \boldsymbol{b}) d \boldsymbol{\theta} .
$$

By substituting $f_{T}(\boldsymbol{\theta} ; \widehat{\boldsymbol{\mu}}, \widehat{\boldsymbol{\Sigma}}, \boldsymbol{a}, \boldsymbol{b})$ with (8), we get:

$$
M(\boldsymbol{v})=\frac{1}{\gamma(2 \pi)^{d / 2}|\widehat{\boldsymbol{\Sigma}}|^{1 / 2}} \int_{\boldsymbol{a}}^{\boldsymbol{b}} \exp \left[-\frac{1}{2}\left\{(\boldsymbol{\theta}-\widehat{\boldsymbol{\mu}})^{T} \widehat{\boldsymbol{\Sigma}}^{-1}(\boldsymbol{\theta}-\widehat{\boldsymbol{\mu}})-2 \boldsymbol{v}^{T} \boldsymbol{\theta}\right\}\right] d \boldsymbol{\theta}
$$

where $\gamma=F(\boldsymbol{b} ; \widehat{\boldsymbol{\mu}}, \widehat{\boldsymbol{\Sigma}})-F(\boldsymbol{a} ; \widehat{\boldsymbol{\mu}}, \widehat{\boldsymbol{\Sigma}})$. The exponent term in (A2) can be extracted separately as $\mathrm{A}(\widehat{\boldsymbol{\mu}})=-\frac{1}{2}\left\{(\boldsymbol{\theta}-\widehat{\boldsymbol{\mu}})^{T} \widehat{\boldsymbol{\Sigma}}^{-1}(\boldsymbol{\theta}-\widehat{\boldsymbol{\mu}})-2 \boldsymbol{v}^{T} \boldsymbol{\theta}\right\}$ and, for $\widehat{\boldsymbol{\mu}}=\mathbf{0}, \mathrm{A}(\mathbf{0})$ can be written as:

$$
\mathrm{A}(\mathbf{0})=\frac{1}{2} \boldsymbol{v}^{T} \widehat{\boldsymbol{\Sigma}} \boldsymbol{v}-\frac{1}{2}\left\{(\boldsymbol{\theta}-\widehat{\boldsymbol{\Sigma}} \boldsymbol{v})^{T} \widehat{\boldsymbol{\Sigma}}^{-1}(\boldsymbol{\theta}-\widehat{\boldsymbol{\Sigma}} \boldsymbol{v})\right\}
$$

Thus, when the location parameter $\widehat{\boldsymbol{\mu}}=\mathbf{0}$, the MGF in (A2) can be simplified using (A3) as:

$$
M_{0}(\boldsymbol{v})=\frac{\exp \left(\frac{1}{2} \boldsymbol{v}^{T} \widehat{\boldsymbol{\Sigma}}^{-1} \boldsymbol{v}\right)}{\gamma(2 \pi)^{d / 2}|\widehat{\boldsymbol{\Sigma}}|^{1 / 2}} \int_{\boldsymbol{a}-\widehat{\boldsymbol{\Sigma}} \boldsymbol{v}}^{\boldsymbol{b}-\widehat{\boldsymbol{\Sigma}} \boldsymbol{v}} \exp \left(-\frac{1}{2} \boldsymbol{\theta}^{T} \widehat{\boldsymbol{\Sigma}}^{-1} \boldsymbol{\theta}\right) d \boldsymbol{\theta},
$$

where $M_{0}(\boldsymbol{v})$ denotes the MGF for $\widehat{\boldsymbol{\mu}}=\mathbf{0}$. Define $\Psi_{\gamma}(\widehat{\boldsymbol{\Sigma}})$ as: 


$$
\Psi_{\gamma}(\widehat{\boldsymbol{\Sigma}})=\left[\int_{\boldsymbol{a}-\widehat{\boldsymbol{\Sigma}} \boldsymbol{v}}^{\boldsymbol{b}-\widehat{\boldsymbol{\Sigma}} \boldsymbol{v}} \exp \left(-\frac{1}{2} \boldsymbol{\theta}^{T} \widehat{\boldsymbol{\Sigma}}^{-1} \boldsymbol{\theta}\right) d \boldsymbol{\theta}\right] /\left\{\gamma(2 \pi)^{\frac{d}{2}}|\widehat{\boldsymbol{\Sigma}}|^{\frac{1}{2}}\right\} .
$$

Then, (A4) can be simplified as $M_{0}(\boldsymbol{v})=\exp \left(\frac{1}{2} \boldsymbol{v}^{T} \widehat{\boldsymbol{\Sigma}}^{-1} \boldsymbol{v}\right) \Psi_{\gamma}(\widehat{\boldsymbol{\Sigma}})$. We can obtain the first moment by taking a partial derivative with respect to $v_{j}$ as:

$$
\frac{\partial M_{0}(\boldsymbol{v})}{\partial v_{j}}=\exp \left(\frac{1}{2} \boldsymbol{v}^{T} \widehat{\boldsymbol{\Sigma}} \boldsymbol{v}\right) \frac{\partial \Psi_{\gamma}(\widehat{\boldsymbol{\Sigma}})}{\partial v_{j}}+\Psi_{\gamma}(\widehat{\boldsymbol{\Sigma}}) \frac{\partial \exp \left(\frac{1}{2} \boldsymbol{v}^{T} \widehat{\boldsymbol{\Sigma}} \boldsymbol{v}\right)}{\partial v_{j}}
$$

In (A6), the partial derivative terms can be written as:

$$
\begin{gathered}
\frac{\partial \exp \left(\frac{1}{2} \boldsymbol{v}^{T} \widehat{\boldsymbol{\Sigma}} \boldsymbol{v}\right)}{\partial v_{j}}=\exp \left(\frac{1}{2} \boldsymbol{v}^{T} \widehat{\boldsymbol{\Sigma}} \boldsymbol{v}\right) \sum_{p=1}^{d} \sigma_{j, p} v_{p} \\
\frac{\partial \Psi_{\gamma}(\widehat{\boldsymbol{\Sigma}})}{\partial v_{j}}=\frac{\partial}{\partial v_{j}} \int_{a_{1}^{*}}^{b_{1}^{*}} \cdots \int_{a_{d}^{*}}^{b_{d}^{*}} f_{T}(\boldsymbol{\theta} ; \widehat{\boldsymbol{\mu}}, \widehat{\boldsymbol{\Sigma}}, \boldsymbol{a}, \boldsymbol{b}) d \boldsymbol{\theta}=\sum_{p=1}^{d} \sigma_{j, p}\left\{\varphi_{p}^{*}\left(a_{p}^{*}\right)-\varphi_{p}\left(b_{p}^{*}\right)\right\},
\end{gathered}
$$

where $a_{j}^{*}=a_{j}-\sum_{p=1}^{d} \sigma_{j, p} v_{p}, b_{j}^{*}=b_{j}-\sum_{p=1}^{d} \sigma_{j, p} v_{p}$, and the function $\varphi_{p}^{*}(x)$ is defined as

$$
\varphi_{p}^{*}(x)=\int_{a_{1}^{*}}^{b_{1}^{*}} \cdots \int_{a_{p-1}^{*}}^{b_{p-1}^{*}} \int_{a_{p+1}^{*}}^{b_{p+1}^{*}} \cdots \int_{a_{d}^{*}}^{b_{d}^{*}} f_{T}\left(x, \boldsymbol{\theta}_{-p}\right) d \boldsymbol{\theta}_{-p}
$$

where $\boldsymbol{\theta}_{-p}$ is a $(d-1)$-dimensional vector that excludes $\theta_{p}$, i.e., $\boldsymbol{\theta}_{-p}=\left[\theta_{1}, \ldots, \theta_{p-1}, \theta_{p+1}, \ldots, \theta_{d}\right]^{T}$. Because, $a_{p}^{*}=a_{p}$ and $b_{p}^{*}=b_{p}$ for $\boldsymbol{v}=\mathbf{0}, \varphi_{p}^{*}(x)$ can be simplified as:

$$
\left.\varphi_{p}^{*}(x)\right|_{v=\mathbf{0}}=\varphi_{p}(x)=\int_{a_{1}}^{b_{1}} \cdots \int_{a_{p-1}}^{b_{p-1}} \int_{a_{p-1}}^{b_{p+1}} \cdots \int_{a_{d}}^{b_{d}} f_{T}\left(x, \boldsymbol{\theta}_{-p}\right) d \boldsymbol{\theta}_{-p} .
$$

From (A7), (A8) and (A10), the first truncated moment now can be computed as:

$$
\left.\frac{\partial M_{0}(\boldsymbol{v})}{\partial v_{j}}\right|_{\boldsymbol{v}=\mathbf{0}}=\sum_{p=1}^{d} \sigma_{j, p}\left\{\varphi_{p}\left(a_{p}\right)-\varphi_{p}\left(b_{p}\right)\right\}
$$

The first moment in (A11) is only valid when the location parameter is $\mathbf{0}$. However, the general first moment with any location parameter can be easily derived as:

$$
\hat{\mu}_{j}^{*}=\left.\frac{\partial M(\boldsymbol{v})}{\partial v_{j}}\right|_{v=0}=\hat{\mu}_{j}+\sum_{p=1}^{d} \sigma_{j, p}\left\{\varphi_{p}\left(a_{p}\right)-\varphi_{p}\left(b_{p}\right)\right\} .
$$

To obtain the second moment, we take partial derivative to (A6) with respect to $v_{p}$ as:

$$
\frac{\partial^{2} M_{0}(\boldsymbol{v})}{\partial v_{p} \partial v_{j}}=e^{\frac{1}{2} v^{T} \widehat{\Sigma} v} \frac{\partial^{2} \Psi_{\gamma}(\widehat{\boldsymbol{\Sigma}})}{\partial v_{p} \partial v_{j}}+\frac{\partial \Psi_{\gamma}(\widehat{\boldsymbol{\Sigma}})}{\partial v_{j}} \frac{\partial e^{\frac{1}{2} v^{T} \widehat{\boldsymbol{\Sigma}} v}}{\partial v_{p}}+\Psi_{\gamma}(\widehat{\boldsymbol{\Sigma}}) \frac{\partial^{2} e^{\frac{1}{2} v^{T} \widehat{\boldsymbol{\Sigma}} v}}{\partial v_{p} \partial v_{j}}+\frac{\partial e^{\frac{1}{2} v^{T} \widehat{\boldsymbol{\Sigma}} v}}{\partial v_{j}} \frac{\partial \Psi_{\gamma}(\widehat{\boldsymbol{\Sigma}})}{\partial v_{p}}
$$

where first derivatives are already obtained in (A7) and (A8). The second derivatives are: 


$$
\begin{gathered}
\frac{\partial^{2} e^{\frac{1}{2} v^{T} \widehat{\boldsymbol{\Sigma}} v}}{\partial v_{p} \partial v_{j}}=\sigma_{j, p} \\
\frac{\partial^{2} \Psi_{\gamma}(\widehat{\boldsymbol{\Sigma}})}{\partial v_{p} \partial v_{j}}=\sum_{q=1}^{d}\left(\sigma_{j, q} \frac{\partial \varphi_{q}^{*}\left(a_{q}^{*}\right)}{\partial v_{p}}\right)-\sum_{q=1}^{d}\left(\sigma_{j, q} \frac{\partial \varphi_{q}^{*}\left(b_{q}^{*}\right)}{\partial v_{p}}\right) .
\end{gathered}
$$

The partial derivative of a one-dimensional marginal density $\varphi_{q}^{*}\left(a_{q}^{*}\right)$ in (A15), i.e., $\partial \varphi_{q}^{*}\left(a_{q}^{*}\right) / \partial v_{p}$ and $\partial \varphi_{q}^{*}\left(b_{q}^{*}\right) / \partial v_{p}$, can be computed by adopting the results in Lee (1979), i.e., partial derivative of a one-dimensional marginal density can be expressed in terms of bivariate marginal densities. Thus, $\partial \varphi_{q}^{*}\left(a_{q}^{*}\right) / \partial v_{p}$ can be expressed as:

$$
\frac{\partial \varphi_{q}^{*}\left(a_{q}^{*}\right)}{\partial v_{p}}=\frac{\sigma_{p, q} a_{q}^{*} \varphi_{q}^{*}\left(a_{q}^{*}\right)}{\sigma_{q, q}}+\sum_{q \neq l}\left(\sigma_{p, l}-\frac{\sigma_{q, l} \sigma_{p, q}}{\sigma_{q, q}}\right)\left\{\varphi_{q, l}^{*}\left(a_{q}^{*}, a_{l}^{*}\right)-\varphi_{q, l}^{*}\left(a_{q}^{*}, b_{l}^{*}\right)\right\},
$$

where $\varphi_{q, l}^{*}(x, y)$ is defined as:

$$
\varphi_{q, l}^{*}(x, y)=\int_{a_{1}^{*}}^{b_{1}^{*}} \cdots \int_{a_{q-1}^{*}}^{b_{q-1}^{*}} \int_{a_{q+1}^{*}}^{b_{q+1}^{*}} \cdots \int_{a_{l-1}^{*}}^{b_{l-1}^{*}} \int_{a_{l+1}^{*}}^{b_{l+1}^{*}} \cdots \int_{a_{d}^{*}}^{b_{d}^{*}} f_{T}\left(x, y, \boldsymbol{\theta}_{-q-l}\right) d \boldsymbol{\theta}_{-q-l},
$$

where $(d-2)$-dimensional vector $\boldsymbol{\theta}_{-q-l}=\left[\theta_{1}, \ldots, \theta_{q-1}, \theta_{q+1}, \ldots, \theta_{l-1}, \theta_{l+1}, \ldots, \theta_{d}\right]^{T}$ for $q \neq l$. As shown in (A17), $\varphi_{q, l}^{*}(x, y)$ is a bivariate marginal density with transformed integration limits defined as $a_{j}^{*}=a_{j}-\sum_{p=1}^{d} \sigma_{j, p} v_{p}$ and $b_{j}^{*}=b_{j}-\sum_{p=1}^{d} \sigma_{j, p} v_{p}$.

From (A14)-(A17), the second moment can be derived as:

$$
\begin{aligned}
\left.\frac{\partial^{2} M_{0}(\boldsymbol{v})}{\partial v_{p} \partial v_{j}}\right|_{v=\mathbf{0}}= & \sigma_{j, p}+\sum_{q=1}^{d} \frac{\sigma_{j, q} \sigma_{p, q}\left\{a_{q} \varphi_{q}\left(a_{q}\right)-b_{q} \varphi_{q}\left(b_{q}\right)\right\}}{\sigma_{q, q}} \\
& +\sum_{q=1}^{d} \sigma_{j, q} \sum_{q \neq l}\left(\sigma_{p, l}-\frac{\sigma_{q, l} \sigma_{p, q}}{\sigma_{q, q}}\right) \\
& \times\left[\left\{\varphi_{q, l}\left(a_{q}, a_{l}\right)-\varphi_{q, l}\left(a_{q}, b_{l}\right)\right\}-\left\{\varphi_{q, l}\left(b_{q}, a_{l}\right)-\varphi_{q, l}\left(b_{q}, b_{l}\right)\right\}\right],
\end{aligned}
$$

where $\varphi_{q, l}(x, y)=\int_{\boldsymbol{\Omega}_{-q-l}} f_{T}\left(x, y, \boldsymbol{\theta}_{-q-l}\right) d \boldsymbol{\theta}_{-q-l}$ because $a_{k}^{*}=a_{k}$ and $b_{k}^{*}=b_{k}$ when $\boldsymbol{v}=\mathbf{0}$. The constrained space is $\boldsymbol{\Omega}_{-q-l}=\left\{a_{j} \leq \theta_{i, j} \leq b_{j}: \forall j \neq q\right.$ and $\left.\forall j \neq l, j=1, \ldots, d\right\}$ for $q \neq l$.

The covariance is defined as:

$$
\operatorname{Cov}^{*}\left[\theta_{j}, \theta_{p}\right]=\left(\left.\frac{\partial^{2} M_{0}(v)}{\partial v_{p} \partial v_{j}}\right|_{v=0}\right)-\left(\left.\frac{\partial M_{0}(v)}{\partial v_{p}}\right|_{v=0}\right)\left(\left.\frac{\partial M_{0}(v)}{\partial v_{j}}\right|_{v=0}\right)
$$

By substituting the first moments derived in (A11) and the second moment in (A18), the covariance can be expressed as: 


$$
\begin{aligned}
\operatorname{Cov}^{*}\left[\theta_{j}, \theta_{p}\right]= & \sigma_{j, p}+\sum_{q=1}^{d} \frac{\sigma_{j, q} \sigma_{p, q}\left\{a_{q} \varphi_{q}\left(a_{q}\right)-b_{q} \varphi_{q}\left(b_{q}\right)\right\}}{\sigma_{q, q}} \\
& +\sum_{q=1}^{d} \sigma_{j, q} \sum_{q \neq l}\left(\sigma_{p, l}-\frac{\sigma_{q, l} \sigma_{p, q}}{\sigma_{q, q}}\right)\left[\left\{\varphi_{q, l}\left(a_{q}, a_{l}\right)-\varphi_{q, l}\left(a_{q}, b_{l}\right)\right\}\right. \\
& \left.-\left\{\varphi_{q, l}\left(b_{q}, a_{l}\right)-\varphi_{q, l}\left(b_{q}, b_{l}\right)\right\}\right] \\
& -\left[\sum_{q=1}^{d} \sigma_{j, q}\left\{\varphi_{q}\left(a_{q}\right)-\varphi_{q}\left(b_{q}\right)\right\}\right]\left[\sum_{l=1}^{d} \sigma_{p, l}\left\{\varphi_{l}\left(a_{l}\right)-\varphi_{l}\left(b_{l}\right)\right\}\right] .
\end{aligned}
$$

The derivation in (A20) is valid for any general location parameters because the covariance is invariant to the location shift. By reorganizing (A12) and (A20), we can obtain (9) and (10). 